
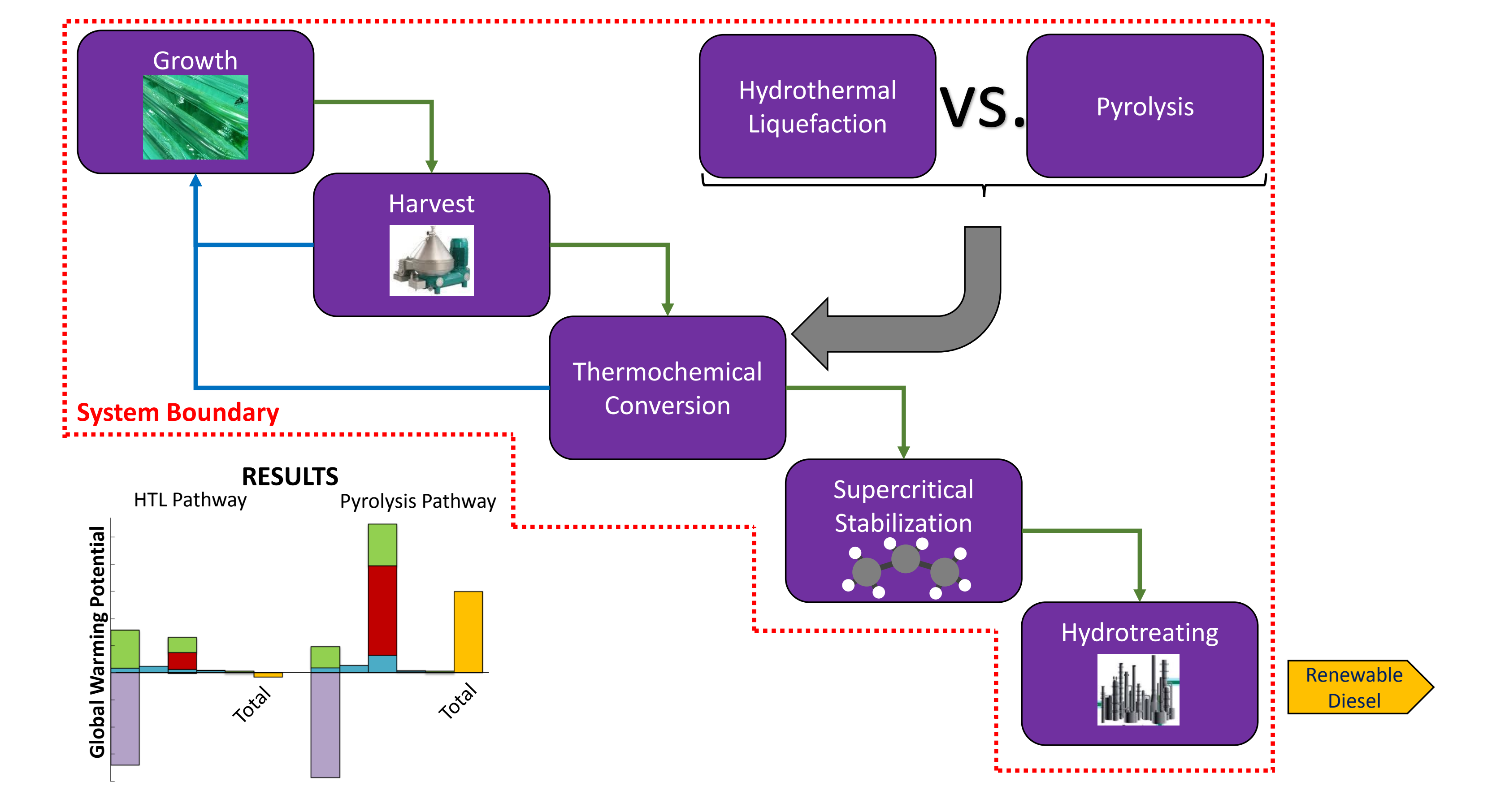


\title{
Lifecycle Assessment of Microalgae to Biofuel: Comparison of Thermochemical Processing Pathways
}

\author{
Edward P. Bennion ${ }^{1}$, Daniel M. Ginosar ${ }^{2}$, John Moses $^{3}$, Foster Agblevor ${ }^{4}$, Jason C. Quinn ${ }^{1 *}$ \\ ${ }^{1}$ Mechanical and Aerospace Engineering Utah State University, Logan, Utah \\ ${ }^{2}$ Biological and Chemistry Processing Department Idaho National Laboratory, Idaho Falls, Idaho \\ ${ }^{3} \mathrm{CF}$ Technologies, Hyde Park, Massachusetts \\ ${ }^{4}$ Biological Engineering, Utah State University, Logan, Utah \\ *corresponding author: 4130 Old Main Hill, Logan, UT 84322-4130, Ph:435-797-0341, \\ email:Jason.Quinn@usu.edu
}

\section{Abstract}

Microalgae is being investigated as a renewable transportation fuel feedstock based on various advantages that include high annual yields, utilization of poor quality land, does not compete with food, and can be integrated with various waste streams. This study focuses on directly assessing the environmental impact of two different thermochemical conversion technologies for the microalgae-to-biofuel process through life cycle assessment. A system boundary of "well to pump" (WTP) is defined and includes sub-process models of the growth, dewatering, thermochemical bio-oil recovery, bio-oil stabilization, conversion to renewable diesel, and transport to the pump. Models were validated with experimental and literature data and are representative of an industrial-scale microalgae-to-biofuel process. Two different thermochemical bio-oil conversion systems are modeled and compared on a systems level, hydrothermal liquefaction (HTL) and pyrolysis. The environmental impact of the two pathways were quantified on the metrics of net energy ratio (NER), defined here as energy consumed over energy produced, and greenhouse gas (GHG) emissions. Results for WTP biofuel production through the HTL pathway were determined to be 1.23 for the NER and GHG emissions of $-11.4 \mathrm{~g} \mathrm{CO}_{2 \text {-eq }}(\mathrm{MJ} \text { renewable diesel })^{-1}$. WTP biofuel production through the pyrolysis pathway results in a NER of 2.27 and $\mathrm{GHG}$ emissions of $210 \mathrm{~g} \mathrm{CO}_{2 \text {-eq }}$ (MJ renewable diesel $)^{-1}$. The large environmental impact associated with the pyrolysis pathway is attributed to feedstock drying requirements and combustion of co-products to improve system energetics. Discussion focuses on a detailed breakdown of the overall process energetics and GHGs, impact of modeling at laboratory-scale compared to industrial-scale, environmental impact sensitivity to systems engineering input parameters for future focused research and development, and a comparison of results to literature. 
35 Abbreviations: Bio-oil Processing (BOP), Carbon Dioxide Equivalence $\left(\mathrm{CO}_{2-\mathrm{eq}}\right)$, Global

36 Warming Potential (GWP), Greenhouse gas (GHG), High Heating Value (HHV),

37 Hydrothermal Liquefaction (HTL), Net Energy Ratio (NER), Life Cycle Assessment

38 (LCA), and Well to Pump (WTP)

40 Key works: Biofuel; Hydrothermal liquefaction; Life Cycle Assessment; Microalgae;

41 Pyrolysis; Thermochemical

\section{Introduction}

The current increase in global energy demand, as well as the negative impact petroleum based energy sources are having on the environment, has led to a renewed interest in renewable energy resources. A variety of third generation feedstocks for biofuel production are being investigated as viable alternatives to traditional energy sources including microalgae based on inherent advantages, specifically characteristically high lipid yields, utilization of poor quality land and water, and integration with point source carbon dioxide sources such as coal fired power plants. Efforts to advance the commercial feasibility of microalgae based biofuels have focused on improvements to the various processing steps associated with the production of feedstock through to fuels. Life cycle assessment (LCA) has emerged as a foundational tool in evaluating alternative processing technologies with results used to highlight areas for further research and development. Various conversion technologies have been identified but the overall impact of the technologies must be understood on a systems level.

In the microalgae to biofuels system there are a variety of conversion technologies being explored in an effort to move towards commercialization. Various technologies have emerged as viable options for the extraction and conversion of biomass to biocrude including but not limited to pyrolysis, hydrothermal liquefaction (HTL), and lipid extraction. Two thermochemical technologies, HTL and pyrolysis, have both been experimentally demonstrated to be viable processes for the conversion of microalgae to bio-oil. Both technologies having the benefit of thermochemically converting non-lipid microalgae constituents into a bio-oil. The HTL conversion process has been demonstrated with a microalgae slurry (microalgae and water mixture), which has the benefit of decreasing the energy requirements for water removal [1-20]. Bio-oil 
recovery through pyrolysis has proven to an effective technology with feedstocks such as

67 woody biomass with limited work on microalgae [2, 21-24]. A challenge that arises with

68 a microalgae feedstock is pyrolysis requires a relatively dry feedstock, $15-20 \%$ moisture

$69[25,26]$. Removal of water to this moisture content requires substantial energy for a

70 microalgae feedstock. Both HTL and pyrolysis have been demonstrated to be feasible

71 with limited assessment on the industrial-scale feasibility of the technologies based on

72 environmental impact [27, 28].

73 LCA has become a premier tool in assessing process energetics and

74 environmental impacts of biofuels production systems. LCAs reported for the microalgae

75 to biofuels process incorporating various conversion technologies have been performed

76 with results varying dramatically due to simplistic process models, differences in

77 production pathways, and incomplete system boundaries [1, 3, 27-58]. The majority of

78 the studies have focused on tradition lipid extraction systems [30, 32, 33, 39, 42, 43, 46,

79 50-53, 55-57, 59]. Only a limited number of studies included assessment of

80 thermochemical conversion technologies on the metrics of net energy and greenhouse gas

81 (GHG) emissions has been limited [1, 27, 28, 34, 60]. Frank et al. [34] examined the

82 environmental impact of an HTL process with a well to pump (WTP) system boundary,

83 but includes an additional processing of HTL byproducts to biogas. de Boer et al. [1]

84 evaluates HTL as a conversion system but fails to include microalgae growth,

85 downstream processing of bio-oil, and HTL byproducts in the analysis. An alternative

86 thermochemical processing technology, pyrolysis, has received minimal evaluation [27].

87 A LCA was carried out by Grierson et al. [27] for a WTP system boundary with the

88 growth system based on a photobioreactor archetecture and spray drying for water

89 removal. These processes are accepted in industry, but are not representative of

90 optimized industrial function. A direct comparison of the energetics of microalgae bio-

91 oil recovery through pyrolysis and HTL has been performed but exclusion of upstream

92 and downstream processing limits the use of results for the comparison to other

93 technologies [2, 27]. For assessing the thermochemical conversion of microalgae

94 biomass through pyrolysis or HTL and directly comparing results to other technologies a

95 LCA that account for all energy and GHG contributions in a WTP system boundary is

96 required. 
Based on the current state of the field there exists a need for the evaluation and comparison of the environmental impact of thermochemical processing technologies applied to the microalgae to biofuels process on a systems level. A modular systems engineering model was constructed including growth, dewatering, bio-oil recovery through HTL or pyrolysis, bio-oil stabilization, bio-oil conversion to renewable diesel, and transport and distribution to consumer pumps to define a system boundary of WTP and validated with experimental and literature data. Two system models were developed: 1) a small-scale model representative of the operation of the experimental systems and 2) an industrial-scale model, validated through experimental and literature data, to assess facility function at commercial scale. All-sub process models were validated with experimental data and integrated into a system model representative of the microalgae to biofuel production processes. Literature data was limited to promising growth and dewatering techniques and bio-oil upgrading in the industrial-scale system with experimental data used for HTL and pyrolysis performance. Environmental impact results are presented on the metrics of net energy ratio (NER) and GHG emissions with sub-processing resolution. Discussion focuses on the impact of modeling at industrialscale, sensitivity to process parameters, and a comparison of results to other conversion technologies based on published literature.

\section{Methods}

A modular systems engineering model, which serves as the foundation of the LCAs, is presented in Figure 1. The systems engineering model includes sub-process models of the growth, dewater, bio-oil recovery through either pyrolysis or HTL, bio-oil stabilization, conversion to renewable diesel, and transport and distribution to the pump. System modeling and validation was performed at two scales: 1) small-scale: which leveraged laboratory based production data and 2) industrial-scale which utilized literature and laboratory data for model validation and is intended to represent industrial function. Industrial-scale modeling work focused on accurately capturing the function of a large-scale facility while incorporating experimental yield and product characterization data from thermochemical conversion experimentation. Compared to the small-scale effort, industrial-scale modeling included utilization of energy recovery and realistic industrial-scale operational data for growth and dewatering processes as would be 


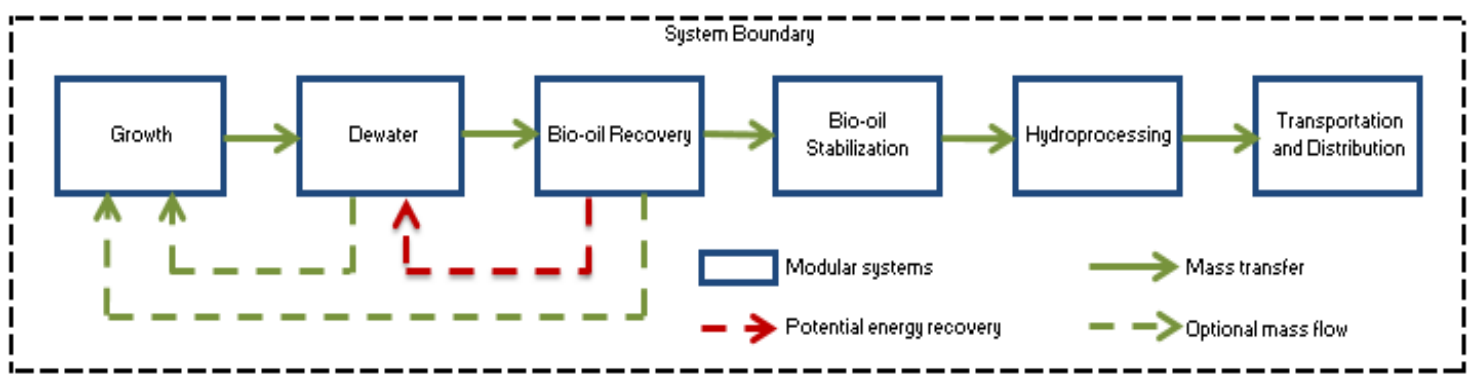

Figure 1: Modular system diagram representative of a 'well to pump' systems boundary for the production of biofuel from microalgae with bio-oil recovery through either pyrolysis or HTL.

Growth and processing facilities are assumed to be co-located to eliminate transportation requirements between processes. The industrial-scaled systems model is the focus of this work, with results for the experimental system presented to illustrate the importance of industrial-scale modeling. The system boundary shown in Figure 1 with bio-oil recovery through HTL or pyrolysis will be referenced to as the "HTL pathway" and the "pyrolysis pathway." Detailed assumptions for each of the sub processes are presented in Table 1.

\subsection{Growth}

The growth system used in cultivation was an open raceway pond located at the

Arizona Center for Microalgae Technology and Innovation growth facility at Arizona State University. Scenedesmus dimorphus was grown in BG-11 medium with macronutrients supplied in the form of laboratory grade $\mathrm{NO}_{3}{ }^{-1}$ and $\mathrm{PO}_{4}{ }^{3-}$ [61]. The system was typically inoculated at $0.5 \mathrm{~g} \mathrm{~L}^{-1}$ and harvested at $1.5 \mathrm{~g} \mathrm{~L}^{-1}$ corresponding to an annual average productivity of $6.5 \mathrm{~g} \mathrm{~m}^{-2} \mathrm{~d}^{-1}$. The produced microalgae biomass was assumed to be $50 \%$ carbon content by weight [62]. Raceway pond circulation was provided through a paddle wheel with an energy consumption of $4.05 \mathrm{MJ}$ (kg microalgae $)^{-1}$. Based on experimental results, dried microalgae before conversion is assumed to have an energy density of $24 \mathrm{MJ} \mathrm{kg}^{-1}$. 
Table 1: System modeling energy and mass inputs for all sub processes in the microalgae to biofuels process

\begin{tabular}{|c|c|c|c|}
\hline Description & $\begin{array}{l}\text { Experimental } \\
\text { System }\end{array}$ & $\begin{array}{l}\text { Industrial-scale } \\
\text { System }\end{array}$ & Units \\
\hline \multicolumn{4}{|l|}{ Microalgae Growth } \\
\hline Microalgae growth rate & 6.5 & 13 & $\mathrm{~g} \mathrm{~m}^{-2} \mathrm{day}^{-1}$ \\
\hline Water losses & $1,082.77$ & $1,082.77$ & $\mathrm{~L}(\mathrm{~kg} \text { microalgae })^{-1}$ \\
\hline \multicolumn{4}{|l|}{ Nutrients } \\
\hline BG-11 & 0.92 & - & $\mathrm{kg}(\mathrm{kg} \text { microalgae })^{-1}$ \\
\hline Urea & - & 0.19 & $\mathrm{~kg}(\mathrm{~kg} \text { microalgae })^{-1}$ \\
\hline Diammonium Phosphate & - & 0.05 & $\mathrm{~kg}(\mathrm{~kg} \text { microalgae })^{-1}$ \\
\hline Growth circulation power & 12.28 & 2.72 & $\mathrm{MJ}(\mathrm{kg} \text { microalgae })^{-1}$ \\
\hline \multicolumn{4}{|l|}{ Dewatering } \\
\hline Dewatering & 11.03 & 0.77 & MJ (kg microalgae $)^{-1}$ \\
\hline Total microalgae mass losses & 15 & 11 & $\%$ \\
\hline \multicolumn{4}{|l|}{ HTL Bio-oil recovery } \\
\hline $\mathrm{NaCO}_{3}$ catalyst & 0.04 & 0.04 & $\mathrm{~kg}(\mathrm{~kg} \text { microalgae })^{-1}$ \\
\hline HTL unit & 6.51 & 6.51 & $\mathrm{MJ}(\mathrm{kg} \text { microalgae })^{-1}$ \\
\hline Energy recovery & - & 0.61 & $\mathrm{MJ}(\mathrm{kg} \text { microalgae })^{-1}$ \\
\hline Heat transfer efficiency & 85 & 85 & $\%$ \\
\hline \multicolumn{4}{|l|}{ Pyrolysis Bio-oil recovery } \\
\hline Freeze drying & 19.01 & - & $\mathrm{MJ}(\mathrm{kg} \text { microalgae })^{-1}$ \\
\hline Rotary drum drying & - & 7.76 & $\mathrm{MJ}(\mathrm{kg} \text { microalgae })^{-1}$ \\
\hline $\mathrm{NaCO}_{3}$ catalyst & 0.027 & 0.027 & $\mathrm{Kg}(\mathrm{kg} \text { microalgae })^{-1}$ \\
\hline Pyrolysis unit & 10.21 & 10.21 & $\mathrm{MJ}(\mathrm{kg} \text { microalgae })^{-1}$ \\
\hline Energy recovered & - & 6.60 & $\mathrm{MJ}(\mathrm{kg} \text { microalgae })^{-1}$ \\
\hline Heat transfer efficiency & 85 & 85 & $\%$ \\
\hline \multicolumn{4}{|l|}{ Bio-oil Stabilization } \\
\hline Processing & 2.15 & 0.77 & MJ (kg Bio-oil $)^{-1}$ \\
\hline Propane losses & 0.02 & 0.02 & $\mathrm{Kg}(\mathrm{kg} \text { Bio-oil })^{-1}$ \\
\hline \multicolumn{4}{|l|}{ Hydroprocessing } \\
\hline Hydrogen & - & 0.0488 & $\mathrm{~kg}(\mathrm{~kg} \text { stable Bio-oil })^{-1}$ \\
\hline Hydrogen production & - & 56.95 & MJ (kg hydrogen $)^{-1}$ \\
\hline Hydroprocessing & - & 0.8381 & MJ (kg stable Bio-oil $)^{-1}$ \\
\hline Zeolite Catalyst & - & 0.0004 & $\operatorname{kg}(\mathrm{kg} \text { stable Bio-oil })^{-1}$ \\
\hline \multicolumn{4}{|l|}{ Transportation and Distribution } \\
\hline Transportation and Distribution & - & 0.34 & $\mathrm{MJ}(\mathrm{kg} \text { renewable diesel })^{-1}$ \\
\hline
\end{tabular}

155 data for the energy requirements and productivity. The industrial-scale system was

156 assumed to produce at a rate of $13 \mathrm{~g} \mathrm{~m}^{-2}$ day $^{-1}$ based on an open raceway pond requiring

$1572.72 \mathrm{MJ}(\mathrm{kg} \text { microalgae })^{-1}$ with a harvest concentration of $0.5 \mathrm{~g} \mathrm{~L}^{-1}[29,34,46-48]$. In

158 the scaled system the carbon, nitrogen and phosphorus ratios remain unchanged from the

159 experimental data. The source of nitrogen is supplied using urea, and the phosphorus is 
160

161

162

163

164

165

166

167

168

169

170

171

172

173

174

175

176

177

178

179

180

181

182

183

184

185

186

187

188

189

supplied through diammonium phosphate as these sources represent economically viable nutrient sources with experimental data supporting microalgae growth on these sources [50,63]. Carbon dioxide is supplied through co-location with an industrial point source, such as coal derived flue gas [64].

\subsection{Dewatering}

The algal concentration after growth in the open raceway pond requires water removal before the biomass can be further processed. In the experimental system excess water was removed using a membrane filtration system which increased the algal concentration from the harvest density of $1.5 \mathrm{~g} \mathrm{~L}^{-1}$ to $40 \mathrm{~g} \mathrm{~L}^{-1}$. A centrifuge was then used to increase the algal concentration to $220 \mathrm{~g} \mathrm{~L}^{-1}$. This concentration is adequate for bio-oil recovery through HTL, but further water must be removed for bio-oil recovery through pyrolysis. In the small-scale experimental system this was done through lyophilizing. Microalgae mass losses in the dewatering sub-process for the experimental system was modeled at $15 \%$.

Industrial-scale system modeling of the dewater system was based on the use of a preliminary bio-flocculation system, used to increase the algal concentration from $0.5 \mathrm{~g}$ $\mathrm{L}^{-1}$ to $10 \mathrm{~g} \mathrm{~L}^{-1}$, followed by dissolved air flotation, to increase algal concentration to $15 \mathrm{~g}$ $\mathrm{L}^{-1}$ and finally a centrifuge for a final concentration of $240 \mathrm{~g} \mathrm{~L}^{-1}[34,49]$. The centrifuge energy requirements and performance is based on an Evodos type 10 centrifuge [65]. A final concentration of approximately $20 \%$ solids is adequate for bio-oil recovery of microalgae to bio-oil through HTL. For pyrolysis bio-oil recovery further dewater was achieved with a rotary drum, which is detailed in the pyrolysis sub process assessment. Microalgae mass losses through the dewatering process from bio flocculation through centrifugation are approximately $11 \%$.

\subsection{Hydrothermal Liquefaction (HTL)}

HTL has been demonstrated to effectively convert wet, $20 \%$ solids, microalgae feedstock into bio-oil $[2,28,34,66]$. Batch experimental data was collected on a reactor operated at $310^{\circ} \mathrm{C}$ and $10,500 \mathrm{kPa}$ with a zeolite catalyst. Products from the HTL bio-oil recovery process include bio-oil, solids, gasses, and an aqueous phase with experimental yields by mass of $37 \%, 16 \%, 30 \%$ and $17 \%$ determined respectively. 
The industrial-scaled system is assumed to be an optimized process in terms of energy recovery with yields based on the experimental data. Energy is recovered through the burning of process gasses used to provide heat to the reactor, and through the bio-oil stream using a heat exchanger, which transfers heat to the incoming feed stream with an efficacy of $85 \%$. A process flow of the modeled industrial-scale HTL system is presented in Figure 2. The aqueous phase contains organic carbon, ammonium, and phosphite which are used to supplement the nutrient demands in microalgae growth. The catalyst and solids are separated from the oil through a centrifuge and reused in the processes.

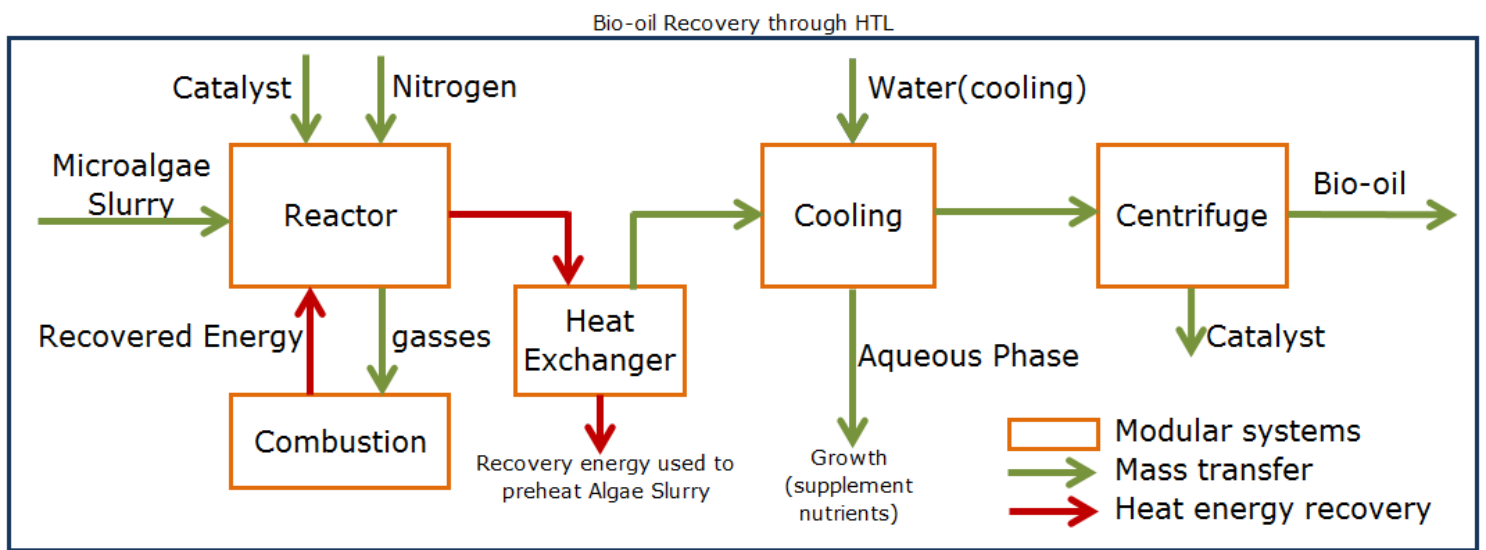

Figure 2: Modular system flow diagram for industrial-scale HTL bio-oil recovery process.

The energetics of the HTL process are dominated by the energy required to heat

204 burning of process gasses. The bio-oil and gasses produced through HTL were energetically favorable process with feedstocks such as switchgrass, soybeans, and wood [67]. A challenge associated with the pyrolysis of algal biomass is the removal of excess

211 water. The microalgae slurry after the dewatering process is $24 \%$ solids and must be

212 further dewatered to $80 \%$ solids prior to pyrolysis processing. In the experimental small-

213 scale model microalgae was dried using freeze drying, $19 \mathrm{MJ}$ (kg microalgae) ${ }^{-1}$, and fed 
214 into the pyrolysis unit reactor at $1,000 \mathrm{~g} \mathrm{hr}^{-1}$ operated with a zeolite catalyst consumed at

215 a rate of $27 \mathrm{mg}$ ( $\mathrm{kg}$ microalgae $)^{-1}$. In the reactor the microalgae feed, gas, and catalyst are

216 heated to $400^{\circ} \mathrm{C}$ and converted into a gas mixture. The gas mixture is then filtered, and

217 cooled before being feed into an electrostatic precipitator where the bio-oil and excess

218 gasses are collected. Products from the pyrolysis process were determined

219 experimentally with mass yields of $29.3 \%, 13.6 \%, 34.3 \%$, and $22.9 \%$ for the bio-oil,

220 char, gasses, and an aqueous phase, respectively.

221 The small-scale experimental results were leveraged for validation of the yield of

222 the industrial-scaled system. Rotary kiln drying operated with natural gas, with an

223 efficiency of $85 \%$ [68], was used in the industrial-scale system to drive off the excess

224 water before pyrolyzing the biomass as it represents an efficient and commercially

225 demonstrated technology [69]. In the industrial-scale system, the pyrolysis reactor

226 energy is supplemented through intersystem energy recovery and combustion of by-

227 products, char and gasses, with HHVs of $25.4 \mathrm{MJ} \mathrm{kg}^{-1}$ and $7.3 \mathrm{MJ} \mathrm{kg}^{-1}$, respectively. A

228 portion of the process gasses are compressed and recycled back into the reactor to

229 maintain an oxygen deprived system. After the pyrolysis process, product gasses from

230 the reactor are filtered and heat is recovered through a heat exchanger with an $85 \%$

231 efficacy. The recovered heat is used to preheat the gas and microalgae mixture as it

232 enters the reactor. A diagram of the industrial-scale system with energy recovery

233 pathways is presented in Figure 3.

234

235

236

237

238
Bio-oil Recovery through Pyrolysis

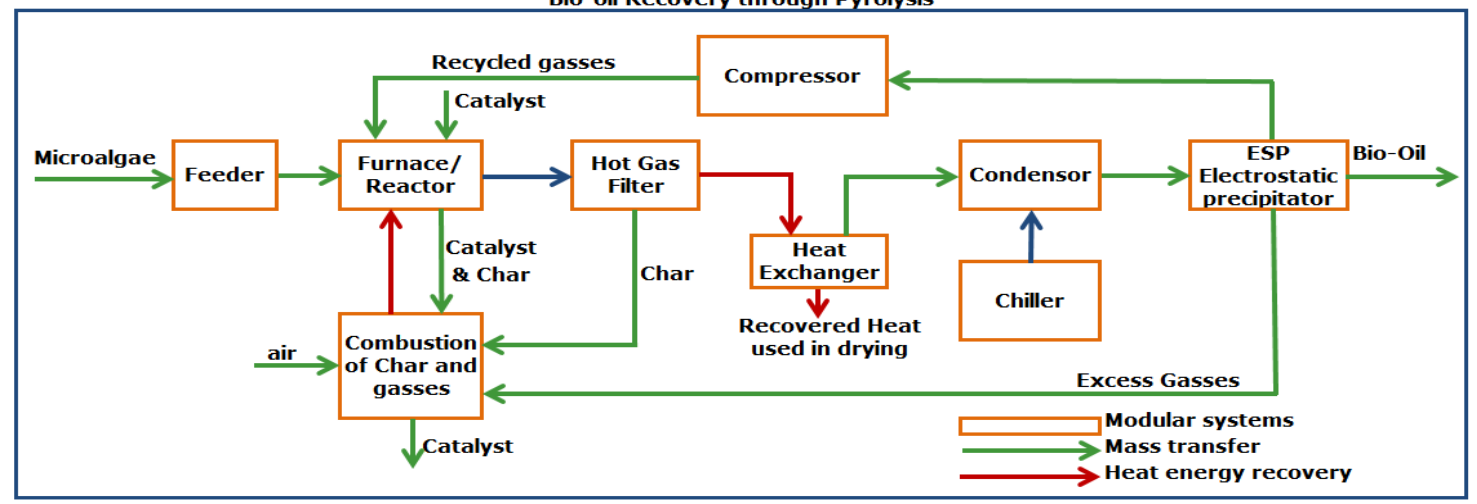

Figure 3: Pyrolysis bio-oil recovery process flow diagram

The pyrolysis sub-process energetic inputs are dominated by the reactor, 7.9 MJ $(\mathrm{kg} \text { microalgae })^{-1}$, and the drying requirements, $7.8 \mathrm{MJ}(\mathrm{kg} \text { microalgae })^{-1}$. Burning of process byproducts are used to supplement the sub-process energetics, supplying 6.6 MJ 
239 (kg microalgae $)^{-1}$. Pyrolysis bio-oil was experimentally determined to have a HHV of

$240 \quad 38.7 \mathrm{MJ} \mathrm{kg}^{-1}$.

241

242

243

244

245

246

247

248

249

250

251

252

253

254

255

256

257

258

259

260

261

262

263

264

265

266

267

268

\subsection{Bio-oil Stabilization Processing}

The bio-oil stabilization process uses near-critical liquid propane to remove unwanted components and stabilize the bio-oil [70]. Stabilization is required due to an increase in the viscosity over time which ultimately results in the bio-oil becoming unusable. The stabilization process is indifferent to upstream thermochemical (HTL or pyrolysis) processing as the biocrudes are assumed to have similar composition. The biooil can be expected to have higher nitrogen and phosphorous content than lignocelluloses feedstocks, however, at least a portion of the $\mathrm{N}$ and $\mathrm{P}$ are removed in the aqueous phase residue from the stabilization process. Any increase in $\mathrm{N}$ and $\mathrm{P}$ content would have a negative impact on downstream refinery processing.

The bio-oil stabilization system incorporates four process steps, an extractor operated at $23^{\circ} \mathrm{C}$ and $3.5 \mathrm{MPa}$ followed by three high pressure separators operated at 3 $\mathrm{MPa}, 2 \mathrm{MPa}$, and 0.2 MPa. Extraction is carried out in a counter-current liquid-liquid extraction column with preheated and pressurized bio-oil entering at the top and near critical propane solvent entering through the bottom at a conservative solvent to feed ratio of five to one. The mixture then flows to the first separator where the pressure is reduced and a portion of the propane is removed. This is repeated through the second and third separator. The pressure is stepped down through the collectors to minimize energy requirements for solvent recycle. Propane that is removed from the first extractor does not require as much energy for recompression before it is recycled back into the extractor, compared to propane that is recovered in the last separator. The solvent is condensed to a liquid state by cooling, and any non-condensable components are purged from the system. The recycle stream is pressurized, reheated, and pumped back to the extractor. Make up solvent is added back to the process to compensate for solvent losses. The bio-oil stabilization process has minimal mass losses, with $15.4 \%$ of the biooil extracted as raffine and $84.6 \%$ extracted as stabilized bio-oil. The energy and material inputs for the bio-oil stabilization process with respect to the experimental and industrialscale system's models are shown in Table 1. The raffine and bio-oil are processed 
269 directly into fuel through hydroprocessing. Further details are presented in the

270 supplementary information.

271

272

273

274

275

276

277

278

279

280

281

282

283

284

285

286

287

288

289

290

291

292

293

294

295

\subsection{Hydroprocessing}

The bio-oil produced through the bio-oil stabilization processing must be further processed to renewable diesel through hydroprocessing, which uses hydrogen to remove excess nitrogen and oxygen from the stabilized bio-oil. The amount of hydrogen needed in hydroprocessing is dependent on the composition of the stabilized bio-oil. The bio-oil composition after stabilization with near-critical liquid propane is shown in Table 2 at two different processing temperatures.

Table 2: Experimental results for pyrolysis bio-oil composition after stabilization processing with near-critical liquid propane

\begin{tabular}{cllll}
\hline Extraction Temperature ${ }^{\circ} \mathrm{C}$ & \%Hydrogen & \%Carbon & \%Nitrogen & \%Oxygen \\
\hline 65 & $8.17 \pm 0.06$ & $50.00 \pm 1.05$ & $0.69 \pm 0.04$ & $41.15 \pm 1.02$ \\
23 & $8.78 \pm 0.22$ & $64.54 \pm 2.08$ & $0.73 \pm 0.03$ & $25.95 \pm 2.28$ \\
\hline
\end{tabular}

Hydrogen demands for hydroprocessing and renewable diesel yields are determined based on the methods of Frank et al. [34] with details presented in the supplementary information. The bio-oil extracted at $23^{\circ} \mathrm{C}$ during the bio-oil stabilization processing yields the best results for hydrogen demands and energy. The bio-oil yields, hydrogen demands, and energy inputs for hydroprocessing were assessed based on the best values found in literature as hydroprocessing was not preformed experimentally.

\section{The hydrogen required and corresponding energy requirement for production for the} processing of the bio-oil is assumed to be derived from natural gas.

The energy requirements for hydroprocessing primarily result from hydrogen production. The processing energy and material inputs are based on a life cycle assessment of corn stover bio-oil with bio-oil recovery through fast pyrolysis [49]. Downstream processing of the corn stover bio-oil includes hydroprocessing which has energy and material inputs that will be roughly the same as those for the stabilized algal bio-oil. Material and energy input for hydroprocessing are shown in Table 1. The bio-oil and raffinate are assumed to have similar properties. 


\subsection{Transportation and Distribution}

Transportation of renewable diesel requires minimal energy and has little impact on the overall energetics of either conversion process, but is included to facilitate comparison to conventional and alternative fuel resources. Energy requirements for transporting renewable diesel are included in Table 1 based on the requirement for soybean based biofuel. It is assumed the production processes are co-located which eliminates the need for transport between sub-processes.

\subsection{Life Cycle Assessment}

Sub-process models focused on accurately capturing energy and mass, for growth, dewater, HTL, pyrolysis, bio-oil stabilization, hydroprocessing, and transportation and distribution were integrated into an engineering system model and serves as the backbone for the LCA. Outputs from the engineering system model serve as the inputs to the LCA modeling. Life cycle inventory (LCI) data was obtained from GREET 2013 and the United States Environmental Protection Agency [71, 72]. The pathways modeled are assessed on two metrics, net energy ratio (NER), and global warming potential (GWP). NER is leveraged as an indicator of the overall energetic effectiveness of the process, equation 1. A NER of less than 1 is desirable with the current NER for conventional petroleum diesel at 0.18 [39].

$$
N E R=\frac{\text { Energy input }}{\text { Energy out in biodiesel }}
$$

The GWP is assessed through the environmental impacts associated with carbon dioxide, methane, and dinitrogen oxide. The three emissions are combined into a carbon dioxide equivalence $\left(\mathrm{CO}_{2 \text {-eq }}\right)$ based on a 100 year GWP of 1, 25, and 298, for carbon dioxide, methane, and dinitrogen oxide, respectively [73]. GWP is detailed for the WTP system boundary of the industrial-scale system for each of the two thermochemical conversion technology pathways modeled. Emissions were separated into three categories: 1) emissions from electrical energy consumption, 2) emissions from production of process heat, and 3) material product consumption. Emissions from product consumption are a result of nutrient demands, system losses, such as losses in catalyst, and burning of process byproducts, such as char and pyrolysis gasses. 


\section{Results and Discussion}

Modular systems engineering models of the microalgae to biofuel process were leveraged to perform a LCA of two different thermochemical conversion pathways at two different scales, small- and industrial-scale. The small-scale system is based on the experimental systems used for process demonstration and evaluation. The industrialscaled system is representative of industrial function through the inclusion of energy recovery through techniques previously discussed, system optimization, and sub-process co-location, and includes experimental results in terms of defining pyrolysis and HTL function.

\subsection{Net Energy, and Greenhouse Gas Emissions}

The NER results for the two different thermochemical processing pathways and modeling scales are broken down by sub-process and presented in Figure 4. The importance of modeling industrial-scale is illustrated in the large difference in NER results for both pathways. The NER for the HTL pathway and pyrolysis pathway are improved by factors of 2.4 and 2.9, respectively, between the small- and industrial-scale modeling efforts. The overall process NER results from the industrial-scale system modeling for HTL and pyrolysis pathways are 1.24 and 2.28, and represent energetically unfavorable systems. In comparison with the NERs of other energy fuels the WTP NERs for conventional diesel, corn ethanol, and soy based biodiesel are $0.18,1.07$, and 0.80 , respectively [39,74].

The energy and material requirements for growth, dewatering, stabilization and hydroprocessing are the same for both pathways evaluated. Slight differences in the subprocess NERs between the two conversion pathways are the result of differences in biooil recovery, oil yields, and heating values as these directly affect the functional units. At the industrial-scale, the HTL pathway has a higher mass yield, 37\%, as compared to the pyrolysis pathway, 29\%. Experimental data showed the HHV in the pyrolysis was $11 \%$ higher than that of the HTL oil. However, the higher bio-oil yield achieved with HTL processing compensates for the lower bio-oil energy density. 


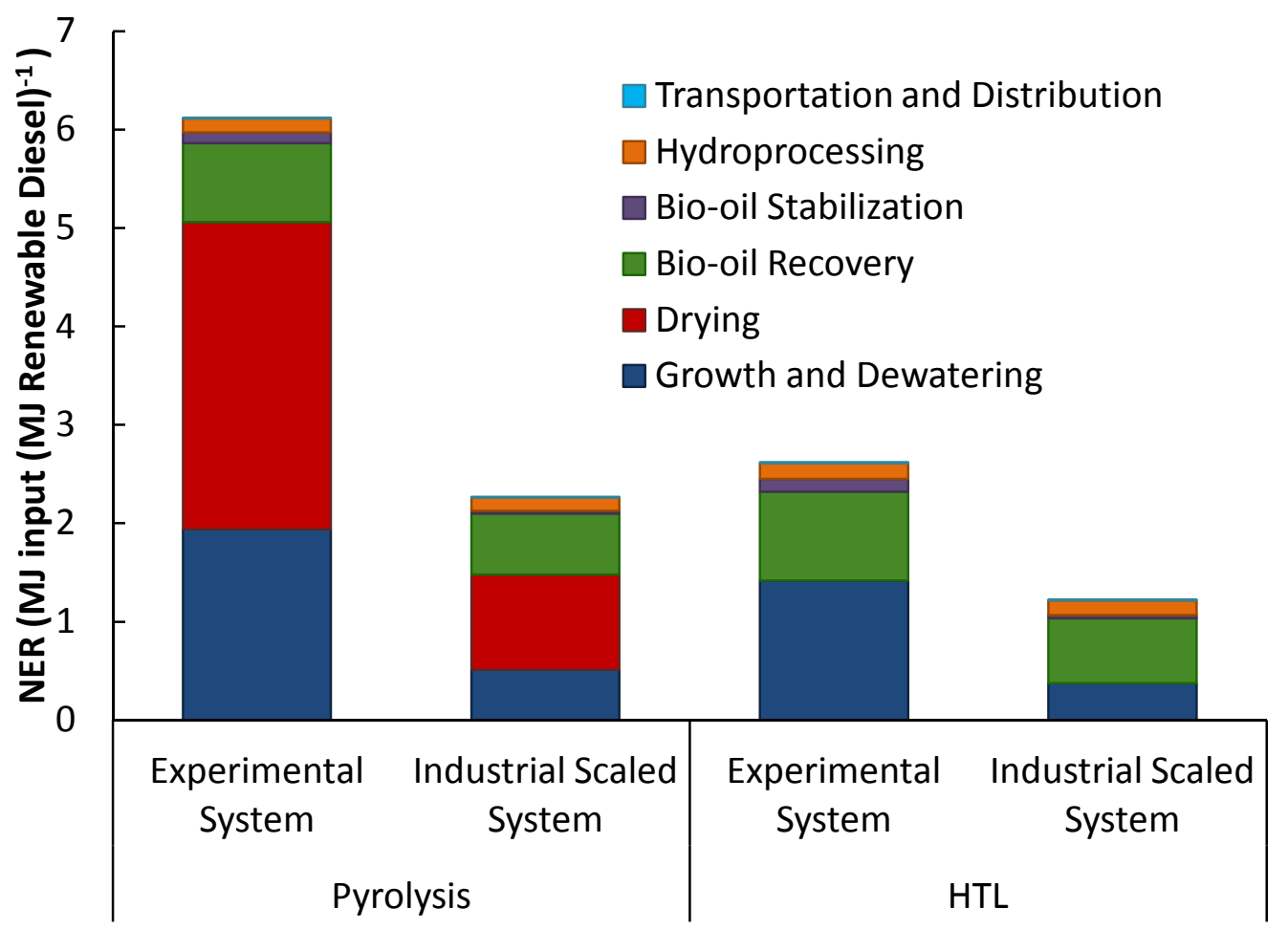

Figure 4: Net Energy Ratio (NER) results for microalgae to renewable diesel with bio-oil recovery through pyrolysis or HTL for small-scale experimental system and the industrial-scaled system.

The results from this study show HTL to be favorable compared to pyrolysis on a system level primarily due to the integration with a wet microalgae slurry (20\% solids), whereas pyrolysis requires dried microalgae ( $80 \%$ solids). The dewater requirements to achieve the percent solids required for HTL conversion facilitates the use of bioflocculation, dissolved air filtration and a centrifuge for removal of water. The pyrolysis pathway requires the remaining water to be removed through thermal methods. Drying of microalgae requires substantial energy, accounting for nearly half (0.97) of the overall NER for the industrial-scale pyrolysis pathway.

The energy flow for the HTL bio-oil recovery processes normalized to 1 unit of energy for the industrial-scale modeling efforts is shown in Figure 5 (A). The HTL process is $55 \%$ efficient in the conversion of embodied feedstock energy to bio-oil. An additional $5.6 \%$ of the sub-process energy is recovered through a heat exchanger and burning of HTL gasses and recycled internally to minimize energy inputs. 
A

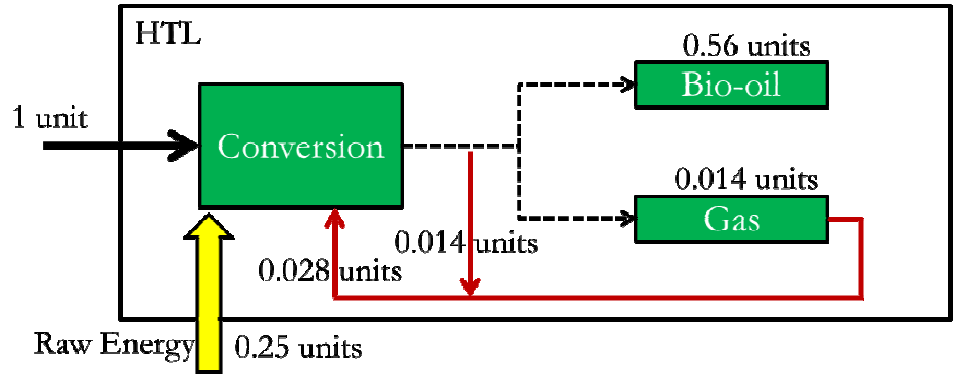

Figure 5: A) Energy flow for the industrial-scale HTL and B) pyrolysis bio-oil recovery sub-processes

371 Comparatively, the pyrolysis sub-process is $51 \%$ efficient in the conversion of

372 embodied feedstock energy to bio-oil, Figure 5 (B). The pyrolysis sub-process is

373 integrated into a bio-refinery system allowing for energy recovery through a heat

374 exchanger and combustion of pyrolysis byproducts, char and gasses. Recovered energy

375 accounts for $28 \%$ of the embodied energy in the feedstock, and is used to supplement the

376 energy demand of the drying unit and heating demands in the reactor. Recovered energy

377 helps the overall energetics of the system, but does not negate the energy demands for

378 drying the microalgae biomass or heating in the reactor. Even with energy recovery the

379 combination of the energy demands in the drying unit and pyrolysis reactor are too large

380 for microalgae conversion through pyrolysis to be made energetically favorable.

\subsection{Global Warming Potential}

383 systems for the two thermochemical conversion technologies modeled and compared to

384 conventional diesel, and soybean based biodiesel, Figure 7. The emissions are broken

385 down into process emissions for electrical, heating, and product consumption. Emissions

386 from product consumption are a result of nutrient demands, material losses, and burning

387 of process byproducts, such as char and pyrolysis or HTL gasses. Extending the boundary

388 to well to wheel (WTW) requires the emissions from the combustion of the fuel assumed 


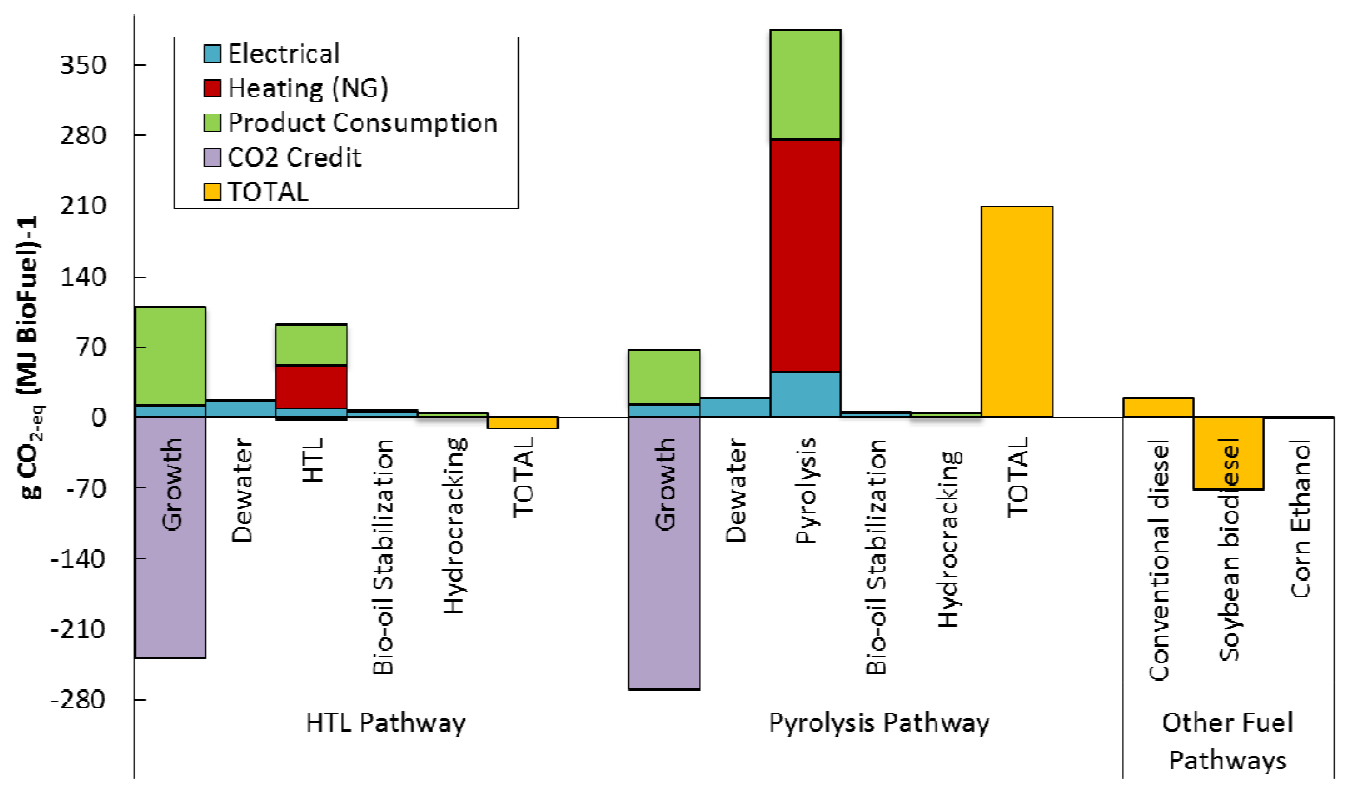

Figure 7: Well to pump GHG emissions for industrial-scale HTL and pyrolysis pathways compared to other traditional fuel pathways $[30,76]$.

Biomass based biofuel production systems benefit from a carbon credit associated with the uptake of carbon dioxide in the growth phase. The GHG emissions for a WTP systems boundary for the HTL pathway results in net negative $\mathrm{CO}_{2 \text {-eq }}$ emissions of -11.4 $\mathrm{g} \mathrm{CO}_{2 \text {-eq }} \mathrm{MJ}^{-1}$. The aqueous phase from the HTL unit contains ammonium and phosphite, which represent a co-product credit, and is assumed to be recycled and supplement the nutrient requirements for microalgae growth. In terms of GWP, a benefit of bio-oil recovery through HTL results from the processing of a wet microalgae slurry, eliminating the energy and GHG emissions associated with drying. In addition, HTL processing produces a small quantity of combustible gases which are burned to improve the energetics of the system. Compared to conventional diesel, the HTL pathway for the production of renewable diesel results in a $32.5 \%$ reduction in GHG emissions based on a WTW system boundary.

Microalgae conversion through the pyrolysis pathway has two energy intensive processes, microalgae drying and heating in the pyrolysis rector which directly impact the environmental impact of the pathway. The reactor energy is supplemented through 
410 detrimental to GHG emissions. If burning of pyrolysis char is replaced with natural gas

411 and the produced char is assumed to be land applied, the GHGs for the production of

412 biofuel are reduced from $210 \mathrm{~g} \mathrm{CO}_{2 \text {-eq }} \mathrm{MJ}^{-1}$ to $166 \mathrm{~g} \mathrm{CO}_{2 \text {-eq }} \mathrm{MJ}^{-1}$, with the NER

413 increasing from 2.28 to 2.63 . Using pyrolysis char for alternative purposes would

414 decrease the environmental impact of the pyrolysis pathway, but GHG emissions are still

415 significantly higher than those of conventional diesel and soy biodiesel, and results in an

416 unfavorable increase in the NER. The need of a dry feedstock and energy demands in the

417 reactor for the pyrolysis unit make it difficult to produce an energetically and

418 environmentally favorable renewable fuel. Emissions from microalgae renewable diesel

419 with pyrolysis are high in comparison with conventional diesel and soybean biodiesel.

420

421

422

423

424

425

426

427

428

429

430

431

432

433

434

\subsection{Sensitivity Analysis}

An assessment of the impact of individual parameters was performed on the industrial-scaled system models to better understand inputs that dramatically affect the energetics and environmental impact of the system. Parameters that had a large impact were revisited for accuracy in the scaled-system modeling to increase certainty in results. Results were also used as feedback to experimental systems to identify areas for improvement. Statistical analysis was performed to identify the critical t-ratio based on a 95\% confidence interval (details presented in supplementary information). The results of the sensitivity analysis for the large-scale microalgae to renewable diesel conversion are shown in Figure 8. Sensitivity results are limited to the top 5 input values shown to have the largest impact with full results presented in the supplementary information.
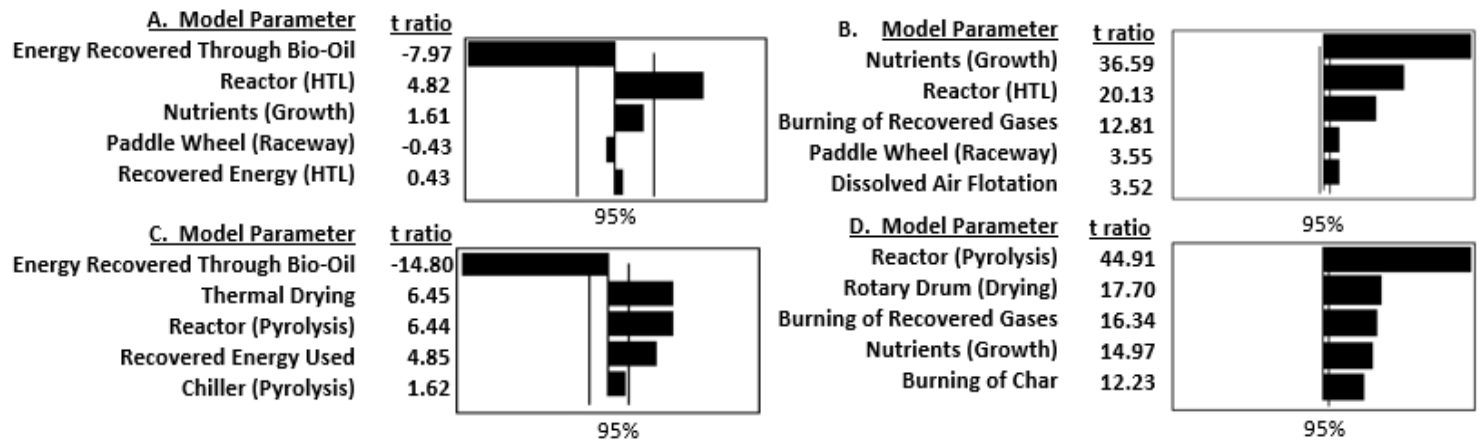

Figure 8: Sensitivity analysis of the WTP system. A) Pyrolysis pathway NER sensitivity, t-critical = \pm 1.73, B) Pyrolysis pathway GHG sensitivity, t-critical $= \pm 1.73$, C) HTL pathway NER sensitivity, tcritical $= \pm 1.75$, D) HTL pathway GHG sensitivity, t-critical $= \pm 1.78$. 
Similarities in the results from the sensitivity analysis for the two thermochemical

436 processes modeled exist as expected. The bio-oil yield represents the functional unit

437 and changes in the yield from the conversion processes will have the largest impact on

438 the system on the metrics of NER. Other inputs shown to be sensitive in the NER

439 sensitivity include reactor energy and recovery of nutrients. Nutrient recycle has been

440 identified as a critical step in the large-scale feasibility of microalgae based biofuel

441 systems [30,77]. For the pyrolysis pathway drying energy and recovery energy are also

442 sensitive as they have a significant impact on the overall process energetics. Sensitivity

443 in GHG emissions for the respective conversion pathways are shown in Figure 8B and

444 8D. Parameters found to be most sensitive in both conversion methods include emissions

445 associated with conversion and emissions associated with growth in the raceway which

446 are primarily a result of nutrient requirements. In the pyrolysis process drying of

447 microalgae and burning of process byproducts were also found to be sensitive.

$448 \quad 3.4$ Comparison with Literature

449 The current immaturity of the microalgae to biofuels processes has led to the

450 evaluation of a variety of processing technologies on the metrics of GWP. LCA

451 facilitates a holistic comparison of individual sub-processes as the work requires

452 considering the entire process from growth to fuel. A comparison of the results in this

453 study was made to other previously published LCA results, Figure 9. The literature

454 survey was limited to studies that report results based on a system boundary consistent

455 with this study, WTP with the conversion methods used in the various studies

456 highlighted. A similar analysis based on the metric of NER is presented in the

457 supplementary information. 


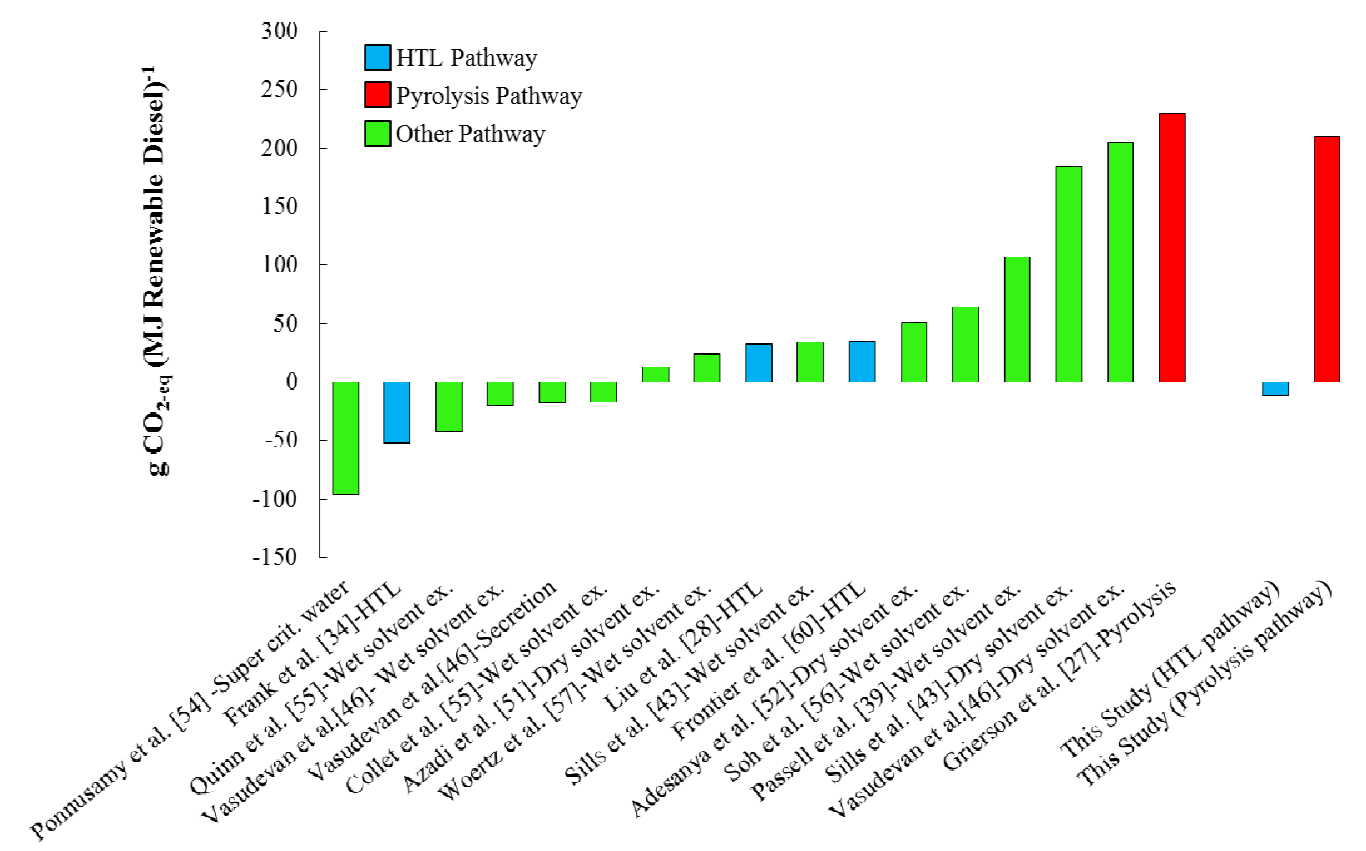

458

459

460

461

462

463

464

465

466

467

468

469

470

471

472

473

474

475

476

477

Figure 9: GHG emissions for microalgae to biofuel with a WTP system boundary as reported in the literature and compared to the results of this study for industrial scale modeling.

The results from the literature survey show a best case WTP GHG emissions for a processes utilizing HTL for conversion of $-52 \mathrm{~g} \mathrm{CO}_{2 \text {-eq }} \mathrm{MJ}^{-1}$ reported by Frank et al. [34]

\section{Two other studies examined conversion through HTL, with GHG emission reported at 33}

$\mathrm{g} \mathrm{CO}_{2 \text {-eq }} \mathrm{MJ}^{-1}$ by Liu et al.[28] and $35.2 \mathrm{~g} \mathrm{CO}_{2 \text {-eq }} \mathrm{MJ}^{-1}$ by Frontier et al. [60]. Differences in processing pathways and end product can be credited with the differences in results of the various HTL based pathways. Frank et al. [34] report a lower GHG result compared to this study which is attributed to differences in downstream processing following biooil recovery through HTL and higher yield. Stabilization and conversion is done through hydrotreating and hydrocracking of HTL bio-oil, while this study uses a near-critical liquid propane stabilization technique followed by hydroprocessing. At current, the estimated yield from hydroprocessing based on the composition of the bio-oil after biooil stabilization processing in this study is $71 \%$. Optimization of this process is expected to increase the efficiency to $90 \%$ which would improve the environmental impact, further harmonizing results. A direct comparison to Frank et al. [34] is presented in the supplementary information that incorporates restricting the system boundary to growth through HTL processing. The higher GHG emissions in Liu et al.[28] compared to this study are the result of differences in processing pathway. Frontier et al. [60] report a 
479 jet fuel compared to renewable diesel in this study. Ultimately, differences in results

480 from the various studies stem from process pathways and assumed HTL performance.

481 Bio-oil production through pyrolysis has been the subject of many studies, but

482 few have evaluated the use of microalgae as the feedstock. In the limited studies that

483 have been performed, differences in pathways require harmonization for direct

484 comparisons. Grierson et al.[27] performs an environmental assessment of a microalgae

485 based biofuel production system incorporating pyrolysis with GHG results reported at

$486290.24 \mathrm{~g} \mathrm{CO}_{2-\mathrm{eq}} \mathrm{MJ}^{-1}$ compared to $210 \mathrm{~g} \mathrm{CO}_{2-\mathrm{eq}} \mathrm{MJ}^{-1}$ from this study. The increased

487 GHG emissions in Grierson et al. [27] is attributed to differences in growth architecture,

488 photobioreactor compared to open raceway pond, and water removal through spray

489 drying compared to rotary drum. Large-scale production systems are expected to operate

490 with a drying system similar to the system used in this effort.

\section{Conclusion}

Microalgae is a promising biofuel feedstock due to its ability to grow on nonarable land, does not compete with food, and high yield. LCA currently is being used to assess the large-scale feasibility and environmental impact of alternative processing technologies being explored for processing microalgae as a feedstock into biofuels. This study integrated experimental and literature data for systems engineering model validation to perform an environmental impact and energetic assessment of two different thermochemical conversion technologies, HTL and pyrolysis. Both conversion pathways result in unfavorable NER results with advances in HTL processing expected to improve energetics. Pyrolysis has proven to be an effective way of converting biomass to a

501 biofuel precursor, however on a systems processing level there are challenges associated 502 with microalgae as a feedstock. The biggest challenge comes from drying the microalgae 503 which represents an energy intensive process. The pyrolysis sub-process with microalgae 504 has the potential to be a self-sustaining process, with the ability to recovery nearly two 505 thirds of the total process energy through heat recovery and the burning of byproducts. 506 Excess energy in the pyrolysis process can be used in other processing steps such as 507 drying. The extra energy is limiting to approximatly $20 \%$ of the energy required in the 508 drying process with the remaining energy derived from natural gas. Results from this 
509 study show the pyrolysis pathway is not energetically or environmentally favorable. This

510 is primarily due to microalgae drying dominating the energetics of the process.

\section{Acknowledgments}

$512 \quad$ Funding was provided by U.S. Department of Energy, Energy Efficiency and

513 Renewable Energy, Bioenergy Technology Office, Project Number 2.13.2.6 Utah State

514 University. Research contributions were provided USU bio lab, INL, ASU, and CF

515 Technologies. The authors acknowledge support from Peter Zemke, Alok Shenoy,

516 Buvanish, Brittany Bennion, and Danna Quinn.

\section{References}

518 [1] de Boer K, Moheimani N, Borowitzka M, Bahri P. Extraction and conversion pathways for

519 microalgae to biodiesel: A review focused on energy consumption. J Appl Phycol. 2012;24:1681-

52098.

521 [2] Vardon DR, Sharma BK, Blazina GV, Rajagopalan K, Strathmann TJ. Thermochemical

522 conversion of raw and defatted algal biomass via hydrothermal liquefaction and slow pyrolysis.

523 Bioresour Technol. 2012;109:178-87.

524 [3] Jones S, Davis R, Zhu Y, Kinchin C, Anderson D, Hallen R, et al. Process design and economics

525 for the conversion of algal biomass to hydrocarbons: Whole algae hydrothermal liquefaction and

526 upgrading. Pasific Northwest National Laboratory; March 2014.

527 [4] López Barreiro D, Prins W, Ronsse F, Brilman W. Hydrothermal liquefaction (htl) of

528 microalgae for biofuel production: State of the art review and future prospects. Biomass

529 Bioenergy. 2013;53:113-27.

530 [5] Biller P, Riley R, Ross AB. Catalytic hydrothermal processing of microalgae: Decomposition 531 and upgrading of lipids. Bioresour Technol. 2011;102:4841-8.

532 [6] Biller P, Ross AB. Potential yields and properties of oil from the hydrothermal liquefaction of 533 microalgae with different biochemical content. Bioresour Technol. 2011;102:215-25.

534 [7] Brown TM, Duan P, Savage PE. Hydrothermal liquefaction and gasification of nannochloropsis 535 sp. Energy and Fuels. 2010;24:3639-46.

536 [8] Dote Y, Sawayama S, Inoue S, Minowa T, Yokoyama S-y. Recovery of liquid fuel from

537 hydrocarbon-rich microalgae by thermochemical liquefaction. Fuel. 1994;73:1855-7.

538 [9] Duan P, Savage PE. Hydrothermal liquefaction of a microalga with heterogeneous catalysts. 539 Industrial and Engineering Chemistry Research. 2011;50:52-61.

540 [10] Garcia Alba L, Torri C, Samorì C, Van Der Spek J, Fabbri D, Kersten SRA, et al. Hydrothermal

541 treatment (htt) of microalgae: Evaluation of the process as conversion method in an algae

542 biorefinery concept. Energy and Fuels. 2012;26:642-57.

543 [11] Jena U, Das KC, Kastner JR. Effect of operating conditions of thermochemical liquefaction on

544 biocrude production from spirulina platensis. Bioresour Technol. 2011;102:6221-9.

545 [12] Matsui T, Nishihara A, Ueda C, Ohtsuki M, Ikenaga N, Suzuki T. Liquefaction of micro-algae 546 with iron catalyst. Fuel. 1997;76:1043-8.

547 [13] Minowa T, Yokoyama S-y, Kishimoto M, Okakura T. Oil production from algal cells of 548 dunaliella tertiolecta by direct thermochemical liquefaction. Fuel. 1995;74:1735-8.

549 [14] Ross AB, Biller P, Kubacki ML, Li H, Lea-Langton A, Jones JM. Hydrothermal processing of

550 microalgae using alkali and organic acids. Fuel. 2010;89:2234-43. 
551

552

553

554

555

556

557

558

559

560

561

562

563

564

565

566

567

568

569

570

571

572

573

574

575

576

577

578

579

580

581

582

583

584

585

586

587

588

589

590

591

592

593

594

595

596

[15] Shuping Z, Yulong W, Mingde Y, Kaleem I, Chun L, Tong J. Production and characterization of bio-oil from hydrothermal liquefaction of microalgae dunaliella tertiolecta cake. Energy. 2010;35:5406-11.

[16] Valdez PJ, Dickinson JG, Savage PE. Characterization of product fractions from hydrothermal liquefaction of nannochloropsis sp. And the influence of solvents. Energy and Fuels. 2011;25:3235-43.

[17] Vardon DR, Sharma BK, Scott J, Yu G, Wang Z, Schideman L, et al. Chemical properties of biocrude oil from the hydrothermal liquefaction of spirulina algae, swine manure, and digested anaerobic sludge. Bioresour Technol. 2011;102:8295-303.

[18] Yang YF, Feng CP, Inamori Y, Maekawa T. Analysis of energy conversion characteristics in liquefaction of algae. Resources, Conservation and Recycling. 2004;43:21-33.

[19] Yu G, Zhang Y, Schideman L, Funk TL, Wang Z. Hydrothermal liquefaction of low lipid content microalgae into bio-crude oil. Transactions of the ASABE. 2011;54:239-46.

[20] Zou S, Wu Y, Yang M, Li C, Tong J. Bio-oil production from sub- and supercritical water liquefaction of microalgae dunaliella tertiolecta and related properties. Energy and Environmental Science. 2010;3:1073-8.

[21] Barta K, Ford PC. Catalytic conversion of nonfood woody biomass solids to organic liquids. Accounts of Chemical Research. 2014;47:1503-12.

[22] Corbetta M, Frassoldati A, Bennadji H, Smith K, Serapiglia MJ, Gauthier G, et al. Pyrolysis of centimeter-scale woody biomass particles: Kinetic modeling and experimental validation. Energy \& Fuels. 2014;28:3884-98.

[23] Septien S, Valin S, Dupont C, Peyrot M, Salvador S. Effect of particle size and temperature on woody biomass fast pyrolysis at high temperature (1000-1400 $\left.{ }^{\circ} \mathrm{C}\right)$. Fuel. 2012;97:202-10.

[24] Wannapeera J, Fungtammasan B, Worasuwannarak N. Effects of temperature and holding time during torrefaction on the pyrolysis behaviors of woody biomass. Journal of Analytical and Applied Pyrolysis. 2011;92:99-105.

[25] Mohan D, Pittman CU, Steele PH. Pyrolysis of wood/biomass for bio-oil: A critical review. Energy \& Fuels. 2006;20:848-89.

[26] Di Blasi C. Modeling chemical and physical processes of wood and biomass pyrolysis.

Progress in Energy and Combustion Science. 2008;34:47-90.

[27] Grierson S, Strezov V, Bengtsson J. Life cycle assessment of a microalgae biomass cultivation, bio-oil extraction and pyrolysis processing regime. Algal Research. 2013;2:299-311.

[28] Liu X, Saydah B, Eranki P, Colosi LM, Greg Mitchell B, Rhodes J, et al. Pilot-scale data provide enhanced estimates of the life cycle energy and emissions profile of algae biofuels produced via hydrothermal liquefaction. Bioresour Technol. 2013;148:163-71.

[29] ANL, NREL, PNNL. Renewable diesel from algal lipids: An integrated baseline for cost, emissions, and resource potential from a harmonized model. US Department of Energy Biomass Program; June 2012.

[30] Batan L, Quinn J, Willson B, Bradley T. Net energy and greenhouse gas emission evaluation of biodiesel derived from microalgae. Environmental Science and Technology. 2010;44:7975-80. [31] Beal CM, Hebner RE, Webber ME, Ruoff RS, Seibert AF. The energy return on investment for algal biocrude: Results for a research production facility. BioEnergy Research. 2012;5:341-62. [32] Brentner LB, Eckelman MJ, Zimmerman JB. Combinatorial life cycle assessment to inform process design of industrial production of algal biodiesel. Environ Sci Technol. 2011;45:7060-7. [33] Campbell PK, Beer T, Batten D. Life cycle assessment of biodiesel production from microalgae in ponds. Bioresour Technol. 2011;102:50-6. 
597 [34] Frank E, Elgowainy A, Han J, Wang Z. Life cycle comparison of hydrothermal liquefaction 598 and lipid extraction pathways to renewable diesel from algae. Mitigation and Adaptation 599 Strategies for Global Change. 2013;18:137-58.

600 [35] Jorquera O, Kiperstok A, Sales EA, Embirucu M, Ghirardi ML. Comparative energy life-cycle 601 analyses of microalgal biomass production in open ponds and photobioreactors. Bioresour 602 Technol. 2010;101:1406-13.

603 [36] Khoo HH, Sharratt PN, Das P, Balasubramanian RK, Naraharisetti PK, Shaik S. Life cycle 604 energy and $\mathrm{co} 2$ analysis of microalgae-to-biodiesel: Preliminary results and comparisons.

605 Bioresour Technol. 2011;102:5800-7.

606 [37] Liu X, Clarens AF, Colosi LM. Algae biodiesel has potential despite inconclusive results to 607 date. Bioresour Technol. 2012;104:803-6.

608 [38] Menger-Krug E, Niederste-Hollenberg J, Hillenbrand T, Hiessl H. Integration of microalgae 609 systems at municipal wastewater treatment plants: Implications for energy and emission 610 balances. Environ Sci Technol. 2012;46:11505-14.

611 [39] Passell H, Dhaliwal H, Reno M, Wu B, Ben Amotz A, Ivry E, et al. Algae biodiesel life cycle 612 assessment using current commercial data. J Environ Manag. 2013;129:103-11.

613 [40] Razon LF, Tan RR. Net energy analysis of the production of biodiesel and biogas from the 614 microalgae: Haematococcus pluvialis and nannochloropsis. Applied Energy. 2011;88:3507-14. 615 [41] Sevigné Itoiz E, Fuentes-Grünewald C, Gasol CM, Garcés E, Alacid E, Rossi S, et al. Energy 616 balance and environmental impact analysis of marine microalgal biomass production for 617 biodiesel generation in a photobioreactor pilot plant. Biomass Bioenergy. 2012;39:324-35. 618 [42] Shirvani T, Yan XY, Inderwildi OR, Edwards PP, King DA. Life cycle energy and greenhouse 619 gas analysis for algae-derived biodiesel. Energy \& Environmental Science. 2011;4:3773-8. 620 [43] Sills DL, Paramita V, Franke MJ, Johnson MC, Akabas TM, Greene CH, et al. Quantitative 621 uncertainty analysis of life cycle assessment for algal biofuel production. Environ Sci Technol. 622 2013;47:687-94.

623 [44] Slade R, Bauen A. Micro-algae cultivation for biofuels: Cost, energy balance, environmental 624 impacts and future prospects. Biomass Bioenergy. 2013;53:29-38.

625 [45] Soratana K, Landis AE. Evaluating industrial symbiosis and algae cultivation from a life cycle 626 perspective. Bioresour Technol. 2011;102:6892-901.

627 [46] Vasudevan V, Stratton RW, Pearlson MN, Jersey GR, Beyene AG, Weissman JC, et al.

628 Environmental performance of algal biofuel technology options. Environ Sci Technol.

629 2012;46:2451-9.

630 [47] Williams PJL, Laurens LML. Microalgae as biodiesel \& biomass feedstocks: Review \& analysis 631 of the biochemistry, energetics \& economics. Energy \& Environmental Science. 2010;3:554-90. 632 [48] Xu LX, Brilman DWF, Withag JAM, Brem G, Kersten S. Assessment of a dry and a wet route 633 for the production of biofuels from microalgae: Energy balance analysis. Bioresour Technol. 634 2011;102:5113-22.

635 [49] Yanan Zhang GH, Robert C Brown. Life cycle assessment of the production of hydrogen and 636 transportation fuels from corn stover via fast pyrolysis. Environmental Research Letters.

$637 \quad 2013 ; 8: 1-13$.

638 [50] Frank ED, Han J, Palou-Rivera I, Elgowainy A, Wang MQ. Life-cycle analysis of algal lipid fuels

639 with the greet model. Oak Ridge, TN: Center for Transportation Research, Energy Systems

640 Division, Argonne National Laboratory; 2011.

641 [51] Azadi P, Brownbridge G, Mosbach S, Smallbone A, Bhave A, Inderwildi O, et al. The carbon 642 footprint and non-renewable energy demand of algae-derived biodiesel. Applied Energy. 643 2014;113:1632-44. 
644 [52] Adesanya VO, Cadena E, Scott SA, Smith AG. Life cycle assessment on microalgal biodiesel 645 production using a hybrid cultivation system. Bioresour Technol. 2014;163:343-55.

646 [53] Collet P, Lardon L, Hélias A, Bricout S, Lombaert-Valot I, Perrier B, et al. Biodiesel from 647 microalgae - life cycle assessment and recommendations for potential improvements.

648 Renewable Energy. 2014;71:525-33.

649 [54] Ponnusamy S, Reddy HK, Muppaneni T, Downes CM, Deng S. Life cycle assessment of 650 biodiesel production from algal bio-crude oils extracted under subcritical water conditions. 651 Bioresour Technol. 2014;170:454-61.

652 [55] Quinn JC, Smith TG, Downes CM, Quinn C. Microalgae to biofuels lifecycle assessment653 multiple pathway evaluation. Algal Research. 2014;4:116-22.

654 [56] Soh L, Montazeri M, Haznedaroglu BZ, Kelly C, Peccia J, Eckelman MJ, et al. Evaluating 655 microalgal integrated biorefinery schemes: Empirical controlled growth studies and life cycle 656 assessment. Bioresour Technol. 2014;151:19-27.

657 [57] Woertz IC, Benemann JR, Du N, Unnasch S, Mendola D, Mitchell BG, et al. Life cycle ghg 658 emissions from microalgal biodiesel - a ca-greet model. Environ Sci Technol. 2014;48:6060-8. 659 [58] Luo D, Hu Z, Choi DG, Thomas VM, Realff MJ, Chance RR. Life cycle energy and greenhouse 660 gas emissions for an ethanol production process based on blue-green algae. Environ Sci Technol. 661 2011;44:8670-7.

662 [59] Baliga R, Powers SE. Sustainable algae biodiesel production in cold climates. International 663 Journal of Chemical Engineering. 2010;4:1-13.

664 [60] Fortier M-OP, Roberts GW, Stagg-Williams SM, Sturm BSM. Life cycle assessment of bio-jet 665 fuel from hydrothermal liquefaction of microalgae. Applied Energy. 2014;122:73-82.

666 [61] Richmond A. Handbook of microalgal culture biotechnology and applied phycology. Oxford, 667 UK: Blackwell Science; 2004.

668 [62] Chisti Y. Biodiesel from microalgae. Biotechnol Adv. 2007;25:294-306.

669 [63] Xin L, Hong-ying H, Ke G, Jia Y. Growth and nutrient removal properties of a freshwater 670 microalga scenedesmus sp. Lx1 under different kinds of nitrogen sources. Ecological Engineering. 671 2010;36:379-81.

672 [64] Quinn JC, Catton KB, Johnson S, Thomas HB. Geographical assessment of microalgae 673 biofuels potential incorporating resource availability. BioEnergy Research. 2012;6:591-600.

674 [65] Evodos B.V. Evodos type 102014.

675 [66] Biddy M, Davis R, Jones S, Zhu Y. Whole algae hydrothermal liquefaction technology 676 pathway. Technical Report. 2013.

677 [67] Fan J, Kalnes TN, Alward M, Klinger J, Sadehvandi A, Shonnard DR. Life cycle assessment of 678 electricity generation using fast pyrolysis bio-oil. Renewable Energy. 2011;36:632-41.

679 [68] Chudnovsky Y. High-efficiency, gas-fired drum dryer for food processing applications. In:

680 Commission CE, editor. PIER Industrial/Agricultural/Water End-Use Energy Efficiency

681 Program2011.

682 [69] Shelef G, Sukenik A, Green M. Microalgae harvesting and processing: A literature review. 683 1984. p. Medium: ED; Size: Pages: 70.

684 [70] Agblevor F, Petkovic, L., Bennion, E. P., Quinn, J. C., Moses, J., Newby, D., Ginosar, D. Bio-oil 685 separation and stabilization by supercritical fluid fractionation - final report. In: Report INL, 686 editor.May 2014.

687 [71] United States Environmental Protection Agency. Direct emissions from stationary 688 combustion sources. May 2008.

689 [72] Argonne National Laboratory. Greet 2013 model. 2013. 
690 [73] Solomon S, Qin D, Manning M, Chen Z, Marquis M, Averyt KB, et al. Contribution of working 691 group $i$ to the fourth assessment report of the intergovernmental panel on climate change, 2007 692 Cambridge University Press; 2007.

693 [74] Murphy D, Hall CS, Powers B. New perspectives on the energy return on (energy)

694 investment (eroi) of corn ethanol. Environment, Development and Sustainability. 2011;13:179-

695202

696 [75] United States Environmental Protection Agency. Average carbon dioxide emissions resulting 697 from gasoline and diesel fuel. 2005.

698 [76] Wang M, Han J, Dunn JB, Cai H, Elgowainy A. Well-to-wheels energy use and greenhouse 699 gas emissions of ethanol from corn, sugarcane and cellulosic biomass for us use. Environmental 700 Research Letters. 2012;7:045905.

701 [77] Pate R, Klise G, Wu B. Resource demand implications for us algae biofuels production scale702 up. Applied Energy. 2011;88:3377-88.

703 


\title{
Lifecycle Assessment of Microalgae to Biofuel: Comparison of Thermochemical Processing Pathways
}

\author{
Edward P. Bennion ${ }^{1}$, Daniel M. Ginosar ${ }^{2}$, John Moses $^{3}$, Foster Agblevor ${ }^{4}$, Jason C. Quinn ${ }^{1 *}$ \\ ${ }^{1}$ Mechanical and Aerospace Engineering Utah State University, Logan, Utah \\ ${ }^{2}$ Biological and Chemistry Processing Department Idaho National Laboratory, Idaho Falls, Idaho \\ ${ }^{3} \mathrm{CF}$ Technologies, Hyde Park, Massachusetts \\ ${ }^{4}$ Biological Engineering, Utah State University, Logan, Utah \\ *corresponding author: 4130 Old Main Hill, Logan, UT 84322-4130, Ph:435-797-0341, \\ email:Jason.Quinn@usu.edu
}

\section{Abstract}

Microalgae is being investigated as a renewable transportation fuel feedstock based on various advantages that include high annual yields, utilization of poor quality land, does not compete with food, and can be integrated with various waste streams. This study focuses on directly assessing the environmental impact of two different thermochemical conversion technologies for the microalgae-to-biofuel process through life cycle assessment. A system boundary of "well to pump" (WTP) is defined and includes sub-process models of the growth, dewatering, thermochemical bio-oil recovery, bio-oil stabilization, conversion to renewable diesel, and transport to the pump. Models were validated with experimental and literature data and are representative of an industrial-scale microalgae-to-biofuel process. Two different thermochemical bio-oil conversion systems are modeled and compared on a systems level, hydrothermal liquefaction (HTL) and pyrolysis. The environmental impact of the two pathways were quantified on the metrics of net energy ratio (NER), defined here as energy consumed over energy produced, and greenhouse gas (GHG) emissions. Results for WTP biofuel production through the HTL pathway were determined to be 1.23 for the NER and GHG emissions of $-11.4 \mathrm{~g} \mathrm{CO}_{2 \text {-eq }}(\mathrm{MJ} \text { renewable diesel })^{-1}$. WTP biofuel production through the pyrolysis pathway results in a NER of 2.27 and GHG emissions of $210 \mathrm{~g} \mathrm{CO}_{2 \text {-eq }}$ (MJ renewable diesel $)^{-1}$. The large environmental impact associated with the pyrolysis pathway is attributed to feedstock drying requirements and combustion of co-products to improve system energetics. Discussion focuses on a detailed breakdown of the overall process energetics and GHGs, impact of modeling at laboratory-scale compared to industrial-scale, environmental impact sensitivity to systems engineering input parameters for future focused research and development, and a comparison of results to literature. 
35 Abbreviations: Bio-oil Processing (BOP), Carbon Dioxide Equivalence $\left(\mathrm{CO}_{2-\mathrm{eq}}\right)$, Global

36 Warming Potential (GWP), Greenhouse gas (GHG), High Heating Value (HHV),

37 Hydrothermal Liquefaction (HTL), Net Energy Ratio (NER), Life Cycle Assessment

38 (LCA), and Well to Pump (WTP)

40 Key works: Biofuel; Hydrothermal liquefaction; Life Cycle Assessment; Microalgae;

41 Pyrolysis; Thermochemical

\section{Introduction}

The current increase in global energy demand, as well as the negative impact petroleum based energy sources are having on the environment, has led to a renewed interest in renewable energy resources. A variety of third generation feedstocks for biofuel production are being investigated as viable alternatives to traditional energy sources including microalgae based on inherent advantages, specifically characteristically high lipid yields, utilization of poor quality land and water, and integration with point source carbon dioxide sources such as coal fired power plants. Efforts to advance the commercial feasibility of microalgae based biofuels have focused on improvements to the various processing steps associated with the production of feedstock through to fuels. Life cycle assessment (LCA) has emerged as a foundational tool in evaluating alternative processing technologies with results used to highlight areas for further research and development. Various conversion technologies have been identified but the overall impact of the technologies must be understood on a systems level.

In the microalgae to biofuels system there are a variety of conversion technologies being explored in an effort to move towards commercialization. Various technologies have emerged as viable options for the extraction and conversion of biomass to biocrude including but not limited to pyrolysis, hydrothermal liquefaction (HTL), and lipid extraction. Two thermochemical technologies, HTL and pyrolysis, have both been experimentally demonstrated to be viable processes for the conversion of microalgae to bio-oil. Both technologies having the benefit of thermochemically converting non-lipid microalgae constituents into a bio-oil. The HTL conversion process has been demonstrated with a microalgae slurry (microalgae and water mixture), which has the benefit of decreasing the energy requirements for water removal [1-20]. Bio-oil 
recovery through pyrolysis has proven to an effective technology with feedstocks such as woody biomass with limited work on microalgae [2, 21-24]. A challenge that arises with a microalgae feedstock is pyrolysis requires a relatively dry feedstock, 15-20\% moisture $[25,26]$. Removal of water to this moisture content requires substantial energy for a microalgae feedstock. Both HTL and pyrolysis have been demonstrated to be feasible with limited assessment on the industrial-scale feasibility of the technologies based on environmental impact $[27,28]$.

LCA has become a premier tool in assessing process energetics and environmental impacts of biofuels production systems. LCAs reported for the microalgae to biofuels process incorporating various conversion technologies have been performed with results varying dramatically due to simplistic process models, differences in production pathways, and incomplete system boundaries [1, 3, 27-58]. The majority of the studies have focused on tradition lipid extraction systems [30, 32, 33, 39, 42, 43, 46, 50-53, 55-57, 59]. Only a limited number of studies included assessment of thermochemical conversion technologies on the metrics of net energy and greenhouse gas

81 (GHG) emissions has been limited [1, 27, 28, 34, 60]. Frank et al. [34] examined the environmental impact of an HTL process with a well to pump (WTP) system boundary, but includes an additional processing of HTL byproducts to biogas. de Boer et al. [1] evaluates HTL as a conversion system but fails to include microalgae growth, downstream processing of bio-oil, and HTL byproducts in the analysis. An alternative thermochemical processing technology, pyrolysis, has received minimal evaluation [27].

87 A LCA was carried out by Grierson et al. [27] for a WTP system boundary with the growth system based on a photobioreactor archetecture and spray drying for water removal. These processes are accepted in industry, but are not representative of optimized industrial function. A direct comparison of the energetics of microalgae biooil recovery through pyrolysis and HTL has been performed but exclusion of upstream and downstream processing limits the use of results for the comparison to other technologies $[2,27]$. For assessing the thermochemical conversion of microalgae

94 biomass through pyrolysis or HTL and directly comparing results to other technologies a

95 LCA that account for all energy and GHG contributions in a WTP system boundary is 96 required. 
Based on the current state of the field there exists a need for the evaluation and comparison of the environmental impact of thermochemical processing technologies applied to the microalgae to biofuels process on a systems level. A modular systems engineering model was constructed including growth, dewatering, bio-oil recovery through HTL or pyrolysis, bio-oil stabilization, bio-oil conversion to renewable diesel, and transport and distribution to consumer pumps to define a system boundary of WTP and validated with experimental and literature data. Two system models were developed: 1) a small-scale model representative of the operation of the experimental systems and 2) an industrial-scale model, validated through experimental and literature data, to assess facility function at commercial scale. All-sub process models were validated with experimental data and integrated into a system model representative of the microalgae to biofuel production processes. Literature data was limited to promising growth and dewatering techniques and bio-oil upgrading in the industrial-scale system with experimental data used for HTL and pyrolysis performance. Environmental impact results are presented on the metrics of net energy ratio (NER) and GHG emissions with sub-processing resolution. Discussion focuses on the impact of modeling at industrialscale, sensitivity to process parameters, and a comparison of results to other conversion technologies based on published literature.

\section{Methods}

A modular systems engineering model, which serves as the foundation of the LCAs, is presented in Figure 1. The systems engineering model includes sub-process models of the growth, dewater, bio-oil recovery through either pyrolysis or HTL, bio-oil stabilization, conversion to renewable diesel, and transport and distribution to the pump. System modeling and validation was performed at two scales: 1) small-scale: which leveraged laboratory based production data and 2) industrial-scale which utilized literature and laboratory data for model validation and is intended to represent industrial function. Industrial-scale modeling work focused on accurately capturing the function of a large-scale facility while incorporating experimental yield and product characterization data from thermochemical conversion experimentation. Compared to the small-scale effort, industrial-scale modeling included utilization of energy recovery and realistic industrial-scale operational data for growth and dewatering processes as would be 


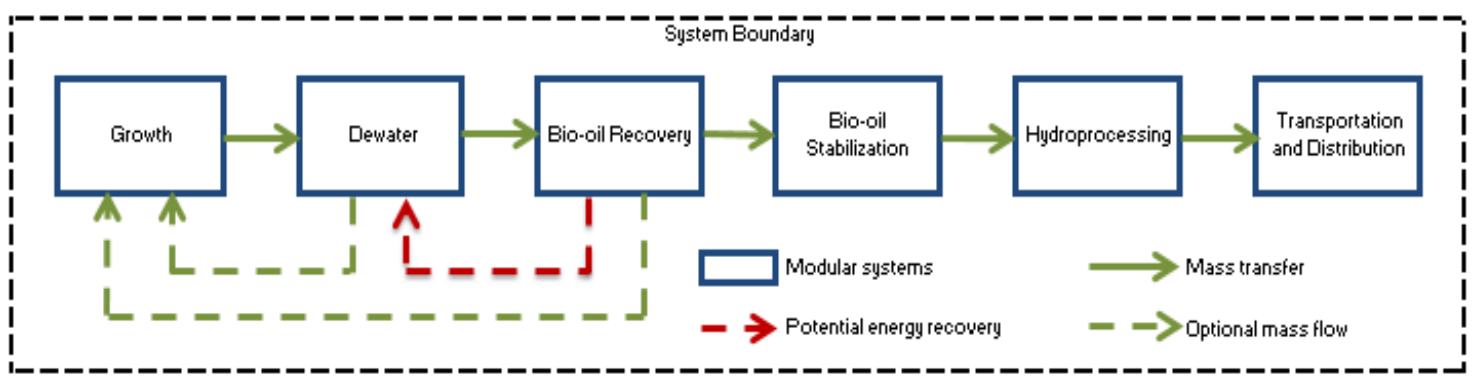

Figure 1: Modular system diagram representative of a 'well to pump' systems boundary for the production of biofuel from microalgae with bio-oil recovery through either pyrolysis or HTL.

Growth and processing facilities are assumed to be co-located to eliminate transportation requirements between processes. The industrial-scaled systems model is the focus of this work, with results for the experimental system presented to illustrate the importance of industrial-scale modeling. The system boundary shown in Figure 1 with bio-oil recovery through HTL or pyrolysis will be referenced to as the "HTL pathway" and the "pyrolysis pathway." Detailed assumptions for each of the sub processes are presented in Table 1.

\subsection{Growth}

The growth system used in cultivation was an open raceway pond located at the

Arizona Center for Microalgae Technology and Innovation growth facility at Arizona State University. Scenedesmus dimorphus was grown in BG-11 medium with macronutrients supplied in the form of laboratory grade $\mathrm{NO}_{3}{ }^{-1}$ and $\mathrm{PO}_{4}{ }^{3-}$ [61]. The system was typically inoculated at $0.5 \mathrm{~g} \mathrm{~L}^{-1}$ and harvested at $1.5 \mathrm{~g} \mathrm{~L}^{-1}$ corresponding to an annual average productivity of $6.5 \mathrm{~g} \mathrm{~m}^{-2} \mathrm{~d}^{-1}$. The produced microalgae biomass was assumed to be $50 \%$ carbon content by weight [62]. Raceway pond circulation was provided through a paddle wheel with an energy consumption of $4.05 \mathrm{MJ}$ (kg microalgae $)^{-1}$. Based on experimental results, dried microalgae before conversion is assumed to have an energy density of $24 \mathrm{MJ} \mathrm{kg}^{-1}$. 
Table 1: System modeling energy and mass inputs for all sub processes in the microalgae to biofuels process

\begin{tabular}{|c|c|c|c|}
\hline Description & $\begin{array}{l}\text { Experimental } \\
\text { System }\end{array}$ & $\begin{array}{l}\text { Industrial-scale } \\
\text { System }\end{array}$ & Units \\
\hline \multicolumn{4}{|l|}{ Microalgae Growth } \\
\hline Microalgae growth rate & 6.5 & 13 & $\mathrm{~g} \mathrm{~m}^{-2} \mathrm{day}^{-1}$ \\
\hline Water losses & $1,082.77$ & $1,082.77$ & $\mathrm{~L}(\mathrm{~kg} \text { microalgae })^{-1}$ \\
\hline \multicolumn{4}{|l|}{ Nutrients } \\
\hline BG-11 & 0.92 & - & $\mathrm{kg}(\mathrm{kg} \text { microalgae })^{-1}$ \\
\hline Urea & - & 0.19 & $\mathrm{~kg}(\mathrm{~kg} \text { microalgae })^{-1}$ \\
\hline Diammonium Phosphate & - & 0.05 & $\mathrm{~kg}(\mathrm{~kg} \text { microalgae })^{-1}$ \\
\hline Growth circulation power & 12.28 & 2.72 & $\mathrm{MJ}(\mathrm{kg} \text { microalgae })^{-1}$ \\
\hline \multicolumn{4}{|l|}{ Dewatering } \\
\hline Dewatering & 11.03 & 0.77 & MJ (kg microalgae $)^{-1}$ \\
\hline Total microalgae mass losses & 15 & 11 & $\%$ \\
\hline \multicolumn{4}{|l|}{ HTL Bio-oil recovery } \\
\hline $\mathrm{NaCO}_{3}$ catalyst & 0.04 & 0.04 & $\mathrm{~kg}(\mathrm{~kg} \text { microalgae })^{-1}$ \\
\hline HTL unit & 6.51 & 6.51 & $\mathrm{MJ}(\mathrm{kg} \text { microalgae })^{-1}$ \\
\hline Energy recovery & - & 0.61 & $\mathrm{MJ}(\mathrm{kg} \text { microalgae })^{-1}$ \\
\hline Heat transfer efficiency & 85 & 85 & $\%$ \\
\hline \multicolumn{4}{|l|}{ Pyrolysis Bio-oil recovery } \\
\hline Freeze drying & 19.01 & - & $\mathrm{MJ}(\mathrm{kg} \text { microalgae })^{-1}$ \\
\hline Rotary drum drying & - & 7.76 & $\mathrm{MJ}(\mathrm{kg} \text { microalgae })^{-1}$ \\
\hline $\mathrm{NaCO}_{3}$ catalyst & 0.027 & 0.027 & $\mathrm{Kg}(\mathrm{kg} \text { microalgae })^{-1}$ \\
\hline Pyrolysis unit & 10.21 & 10.21 & $\mathrm{MJ}(\mathrm{kg} \text { microalgae })^{-1}$ \\
\hline Energy recovered & - & 6.60 & $\mathrm{MJ}(\mathrm{kg} \text { microalgae })^{-1}$ \\
\hline Heat transfer efficiency & 85 & 85 & $\%$ \\
\hline \multicolumn{4}{|l|}{ Bio-oil Stabilization } \\
\hline Processing & 2.15 & 0.77 & MJ (kg Bio-oil $)^{-1}$ \\
\hline Propane losses & 0.02 & 0.02 & $\mathrm{Kg}(\mathrm{kg} \text { Bio-oil })^{-1}$ \\
\hline \multicolumn{4}{|l|}{ Hydroprocessing } \\
\hline Hydrogen & - & 0.0488 & $\mathrm{~kg}(\mathrm{~kg} \text { stable Bio-oil })^{-1}$ \\
\hline Hydrogen production & - & 56.95 & MJ (kg hydrogen $)^{-1}$ \\
\hline Hydroprocessing & - & 0.8381 & MJ (kg stable Bio-oil $)^{-1}$ \\
\hline Zeolite Catalyst & - & 0.0004 & $\operatorname{kg}(\mathrm{kg} \text { stable Bio-oil })^{-1}$ \\
\hline \multicolumn{4}{|l|}{ Transportation and Distribution } \\
\hline Transportation and Distribution & - & 0.34 & $\mathrm{MJ}(\mathrm{kg} \text { renewable diesel })^{-1}$ \\
\hline
\end{tabular}

155 data for the energy requirements and productivity. The industrial-scale system was

156 assumed to produce at a rate of $13 \mathrm{~g} \mathrm{~m}^{-2}$ day $^{-1}$ based on an open raceway pond requiring

$1572.72 \mathrm{MJ}(\mathrm{kg} \text { microalgae })^{-1}$ with a harvest concentration of $0.5 \mathrm{~g} \mathrm{~L}^{-1}[29,34,46-48]$. In

158 the scaled system the carbon, nitrogen and phosphorus ratios remain unchanged from the

159 experimental data. The source of nitrogen is supplied using urea, and the phosphorus is 
160

161

162

163

164

165

166

167

168

169

170

171

172

173

174

175

176

177

178

179

180

181

182

183

184

185

186

187

188

189

supplied through diammonium phosphate as these sources represent economically viable nutrient sources with experimental data supporting microalgae growth on these sources [50,63]. Carbon dioxide is supplied through co-location with an industrial point source, such as coal derived flue gas [64].

\subsection{Dewatering}

The algal concentration after growth in the open raceway pond requires water removal before the biomass can be further processed. In the experimental system excess water was removed using a membrane filtration system which increased the algal concentration from the harvest density of $1.5 \mathrm{~g} \mathrm{~L}^{-1}$ to $40 \mathrm{~g} \mathrm{~L}^{-1}$. A centrifuge was then used to increase the algal concentration to $220 \mathrm{~g} \mathrm{~L}^{-1}$. This concentration is adequate for bio-oil recovery through HTL, but further water must be removed for bio-oil recovery through pyrolysis. In the small-scale experimental system this was done through lyophilizing. Microalgae mass losses in the dewatering sub-process for the experimental system was modeled at $15 \%$.

Industrial-scale system modeling of the dewater system was based on the use of a preliminary bio-flocculation system, used to increase the algal concentration from $0.5 \mathrm{~g}$ $\mathrm{L}^{-1}$ to $10 \mathrm{~g} \mathrm{~L}^{-1}$, followed by dissolved air flotation, to increase algal concentration to $15 \mathrm{~g}$ $\mathrm{L}^{-1}$ and finally a centrifuge for a final concentration of $240 \mathrm{~g} \mathrm{~L}^{-1}[34,49]$. The centrifuge energy requirements and performance is based on an Evodos type 10 centrifuge [65]. A final concentration of approximately $20 \%$ solids is adequate for bio-oil recovery of microalgae to bio-oil through HTL. For pyrolysis bio-oil recovery further dewater was achieved with a rotary drum, which is detailed in the pyrolysis sub process assessment. Microalgae mass losses through the dewatering process from bio flocculation through centrifugation are approximately $11 \%$.

\subsection{Hydrothermal Liquefaction (HTL)}

HTL has been demonstrated to effectively convert wet, $20 \%$ solids, microalgae feedstock into bio-oil $[2,28,34,66]$. Batch experimental data was collected on a reactor operated at $310^{\circ} \mathrm{C}$ and $10,500 \mathrm{kPa}$ with a zeolite catalyst. Products from the HTL bio-oil recovery process include bio-oil, solids, gasses, and an aqueous phase with experimental yields by mass of $37 \%, 16 \%, 30 \%$ and $17 \%$ determined respectively. 
The industrial-scaled system is assumed to be an optimized process in terms of energy recovery with yields based on the experimental data. Energy is recovered through the burning of process gasses used to provide heat to the reactor, and through the bio-oil stream using a heat exchanger, which transfers heat to the incoming feed stream with an efficacy of $85 \%$. A process flow of the modeled industrial-scale HTL system is presented in Figure 2. The aqueous phase contains organic carbon, ammonium, and phosphite which are used to supplement the nutrient demands in microalgae growth. The catalyst and solids are separated from the oil through a centrifuge and reused in the processes.

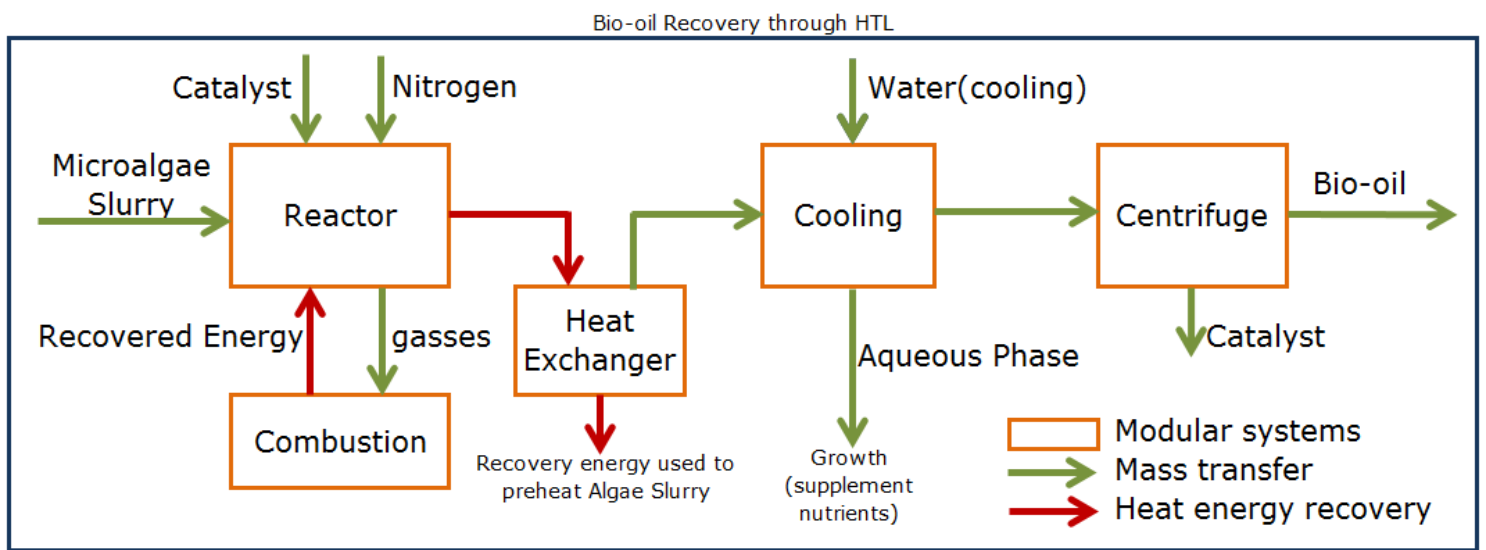

Figure 2: Modular system flow diagram for industrial-scale HTL bio-oil recovery process.

The energetics of the HTL process are dominated by the energy required to heat

204 burning of process gasses. The bio-oil and gasses produced through HTL were energetically favorable process with feedstocks such as switchgrass, soybeans, and wood [67]. A challenge associated with the pyrolysis of algal biomass is the removal of excess

211 water. The microalgae slurry after the dewatering process is $24 \%$ solids and must be

212 further dewatered to $80 \%$ solids prior to pyrolysis processing. In the experimental small-

213 scale model microalgae was dried using freeze drying, $19 \mathrm{MJ}$ (kg microalgae) ${ }^{-1}$, and fed 
214 into the pyrolysis unit reactor at $1,000 \mathrm{~g} \mathrm{hr}^{-1}$ operated with a zeolite catalyst consumed at

215 a rate of $27 \mathrm{mg}$ ( $\mathrm{kg}$ microalgae $)^{-1}$. In the reactor the microalgae feed, gas, and catalyst are

216 heated to $400^{\circ} \mathrm{C}$ and converted into a gas mixture. The gas mixture is then filtered, and

217 cooled before being feed into an electrostatic precipitator where the bio-oil and excess

218 gasses are collected. Products from the pyrolysis process were determined

219 experimentally with mass yields of $29.3 \%, 13.6 \%, 34.3 \%$, and $22.9 \%$ for the bio-oil,

220 char, gasses, and an aqueous phase, respectively.

221 The small-scale experimental results were leveraged for validation of the yield of

222 the industrial-scaled system. Rotary kiln drying operated with natural gas, with an

223 efficiency of 85\% [68], was used in the industrial-scale system to drive off the excess

224 water before pyrolyzing the biomass as it represents an efficient and commercially

225 demonstrated technology [69]. In the industrial-scale system, the pyrolysis reactor

226 energy is supplemented through intersystem energy recovery and combustion of by-

227 products, char and gasses, with HHVs of $25.4 \mathrm{MJ} \mathrm{kg}^{-1}$ and $7.3 \mathrm{MJ} \mathrm{kg}^{-1}$, respectively. A

228 portion of the process gasses are compressed and recycled back into the reactor to

229 maintain an oxygen deprived system. After the pyrolysis process, product gasses from

230 the reactor are filtered and heat is recovered through a heat exchanger with an $85 \%$

231 efficacy. The recovered heat is used to preheat the gas and microalgae mixture as it

232 enters the reactor. A diagram of the industrial-scale system with energy recovery

233 pathways is presented in Figure 3.

234

235

236

237

238
Bio-oil Recovery through Pyrolysis

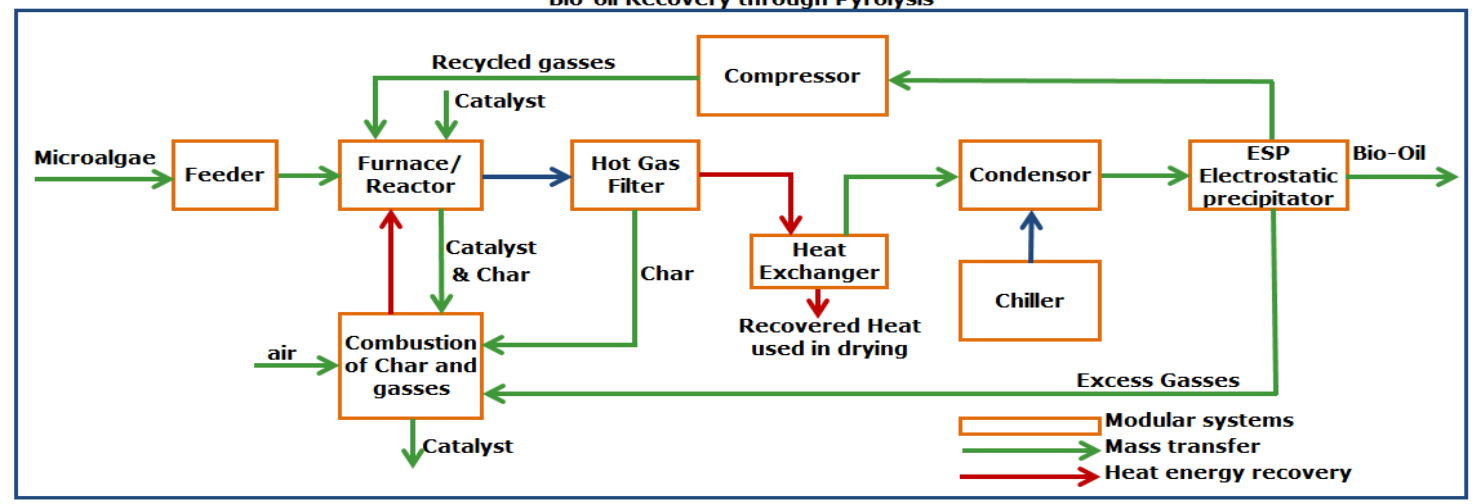

Figure 3: Pyrolysis bio-oil recovery process flow diagram

The pyrolysis sub-process energetic inputs are dominated by the reactor, 7.9 MJ $(\mathrm{kg} \text { microalgae })^{-1}$, and the drying requirements, $7.8 \mathrm{MJ}$ (kg microalgae $)^{-1}$. Burning of process byproducts are used to supplement the sub-process energetics, supplying 6.6 MJ 
239 (kg microalgae $)^{-1}$. Pyrolysis bio-oil was experimentally determined to have a HHV of

$240 \quad 38.7 \mathrm{MJ} \mathrm{kg}^{-1}$.

241

242

243

244

245

246

247

248

249

250

251

252

253

254

255

256

257

258

259

260

261

262

263

264

265

266

267

268

\subsection{Bio-oil Stabilization Processing}

The bio-oil stabilization process uses near-critical liquid propane to remove unwanted components and stabilize the bio-oil [70]. Stabilization is required due to an increase in the viscosity over time which ultimately results in the bio-oil becoming unusable. The stabilization process is indifferent to upstream thermochemical (HTL or pyrolysis) processing as the biocrudes are assumed to have similar composition. The biooil can be expected to have higher nitrogen and phosphorous content than lignocelluloses feedstocks, however, at least a portion of the $\mathrm{N}$ and $\mathrm{P}$ are removed in the aqueous phase residue from the stabilization process. Any increase in $\mathrm{N}$ and $\mathrm{P}$ content would have a negative impact on downstream refinery processing.

The bio-oil stabilization system incorporates four process steps, an extractor operated at $23^{\circ} \mathrm{C}$ and $3.5 \mathrm{MPa}$ followed by three high pressure separators operated at 3 $\mathrm{MPa}, 2 \mathrm{MPa}$, and 0.2 $\mathrm{MPa}$. Extraction is carried out in a counter-current liquid-liquid extraction column with preheated and pressurized bio-oil entering at the top and near critical propane solvent entering through the bottom at a conservative solvent to feed ratio of five to one. The mixture then flows to the first separator where the pressure is reduced and a portion of the propane is removed. This is repeated through the second and third separator. The pressure is stepped down through the collectors to minimize energy requirements for solvent recycle. Propane that is removed from the first extractor does not require as much energy for recompression before it is recycled back into the extractor, compared to propane that is recovered in the last separator. The solvent is condensed to a liquid state by cooling, and any non-condensable components are purged from the system. The recycle stream is pressurized, reheated, and pumped back to the extractor. Make up solvent is added back to the process to compensate for solvent losses. The bio-oil stabilization process has minimal mass losses, with $15.4 \%$ of the biooil extracted as raffine and $84.6 \%$ extracted as stabilized bio-oil. The energy and material inputs for the bio-oil stabilization process with respect to the experimental and industrialscale system's models are shown in Table 1. The raffine and bio-oil are processed 
269 directly into fuel through hydroprocessing. Further details are presented in the

270 supplementary information.

271

272

273

274

275

276

277

278

279

280

281

282

283

284

285

286

287

288

\subsection{Hydroprocessing}

The bio-oil produced through the bio-oil stabilization processing must be further processed to renewable diesel through hydroprocessing, which uses hydrogen to remove excess nitrogen and oxygen from the stabilized bio-oil. The amount of hydrogen needed in hydroprocessing is dependent on the composition of the stabilized bio-oil. The bio-oil composition after stabilization with near-critical liquid propane is shown in Table 2 at two different processing temperatures.

Table 2: Experimental results for pyrolysis bio-oil composition after stabilization processing with near-critical liquid propane

\begin{tabular}{cllll}
\hline Extraction Temperature ${ }^{\circ} \mathrm{C}$ & \%Hydrogen & \%Carbon & \%Nitrogen & \%Oxygen \\
\hline 65 & $8.17 \pm 0.06$ & $50.00 \pm 1.05$ & $0.69 \pm 0.04$ & $41.15 \pm 1.02$ \\
23 & $8.78 \pm 0.22$ & $64.54 \pm 2.08$ & $0.73 \pm 0.03$ & $25.95 \pm 2.28$ \\
\hline
\end{tabular}

Hydrogen demands for hydroprocessing and renewable diesel yields are determined based on the methods of Frank et al. [34] with details presented in the supplementary information. The bio-oil extracted at $23^{\circ} \mathrm{C}$ during the bio-oil stabilization processing yields the best results for hydrogen demands and energy. The bio-oil yields, hydrogen demands, and energy inputs for hydroprocessing were assessed based on the best values found in literature as hydroprocessing was not preformed experimentally. The hydrogen required and corresponding energy requirement for production for the processing of the bio-oil is assumed to be derived from natural gas.

The energy requirements for hydroprocessing primarily result from hydrogen production. The processing energy and material inputs are based on a life cycle assessment of corn stover bio-oil with bio-oil recovery through fast pyrolysis [49]. Downstream processing of the corn stover bio-oil includes hydroprocessing which has energy and material inputs that will be roughly the same as those for the stabilized algal bio-oil. Material and energy input for hydroprocessing are shown in Table 1. The bio-oil and raffinate are assumed to have similar properties. 


\subsection{Transportation and Distribution}

Transportation of renewable diesel requires minimal energy and has little impact on the overall energetics of either conversion process, but is included to facilitate comparison to conventional and alternative fuel resources. Energy requirements for transporting renewable diesel are included in Table 1 based on the requirement for soybean based biofuel. It is assumed the production processes are co-located which eliminates the need for transport between sub-processes.

\subsection{Life Cycle Assessment}

Sub-process models focused on accurately capturing energy and mass, for growth, dewater, HTL, pyrolysis, bio-oil stabilization, hydroprocessing, and transportation and distribution were integrated into an engineering system model and serves as the backbone for the LCA. Outputs from the engineering system model serve as the inputs to the LCA modeling. Life cycle inventory (LCI) data was obtained from GREET 2013 and the United States Environmental Protection Agency [71, 72]. The pathways modeled are assessed on two metrics, net energy ratio (NER), and global warming potential (GWP). NER is leveraged as an indicator of the overall energetic effectiveness of the process, equation 1. A NER of less than 1 is desirable with the current NER for conventional petroleum diesel at 0.18 [39].

$$
N E R=\frac{\text { Energy input }}{\text { Energy out in biodiesel }}
$$

The GWP is assessed through the environmental impacts associated with carbon dioxide, methane, and dinitrogen oxide. The three emissions are combined into a carbon dioxide equivalence $\left(\mathrm{CO}_{2 \text {-eq }}\right)$ based on a 100 year GWP of 1, 25, and 298, for carbon dioxide, methane, and dinitrogen oxide, respectively [73]. GWP is detailed for the WTP system boundary of the industrial-scale system for each of the two thermochemical conversion technology pathways modeled. Emissions were separated into three categories: 1) emissions from electrical energy consumption, 2) emissions from production of process heat, and 3) material product consumption. Emissions from product consumption are a result of nutrient demands, system losses, such as losses in catalyst, and burning of process byproducts, such as char and pyrolysis gasses. 


\section{Results and Discussion}

Modular systems engineering models of the microalgae to biofuel process were leveraged to perform a LCA of two different thermochemical conversion pathways at two different scales, small- and industrial-scale. The small-scale system is based on the experimental systems used for process demonstration and evaluation. The industrialscaled system is representative of industrial function through the inclusion of energy recovery through techniques previously discussed, system optimization, and sub-process co-location, and includes experimental results in terms of defining pyrolysis and HTL function.

\subsection{Net Energy, and Greenhouse Gas Emissions}

The NER results for the two different thermochemical processing pathways and modeling scales are broken down by sub-process and presented in Figure 4. The importance of modeling industrial-scale is illustrated in the large difference in NER results for both pathways. The NER for the HTL pathway and pyrolysis pathway are improved by factors of 2.4 and 2.9, respectively, between the small- and industrial-scale modeling efforts. The overall process NER results from the industrial-scale system modeling for HTL and pyrolysis pathways are 1.24 and 2.28, and represent energetically unfavorable systems. In comparison with the NERs of other energy fuels the WTP NERs for conventional diesel, corn ethanol, and soy based biodiesel are $0.18,1.07$, and 0.80 , respectively [39,74].

The energy and material requirements for growth, dewatering, stabilization and hydroprocessing are the same for both pathways evaluated. Slight differences in the subprocess NERs between the two conversion pathways are the result of differences in biooil recovery, oil yields, and heating values as these directly affect the functional units. At the industrial-scale, the HTL pathway has a higher mass yield, 37\%, as compared to the pyrolysis pathway, 29\%. Experimental data showed the HHV in the pyrolysis was $11 \%$ higher than that of the HTL oil. However, the higher bio-oil yield achieved with HTL processing compensates for the lower bio-oil energy density. 


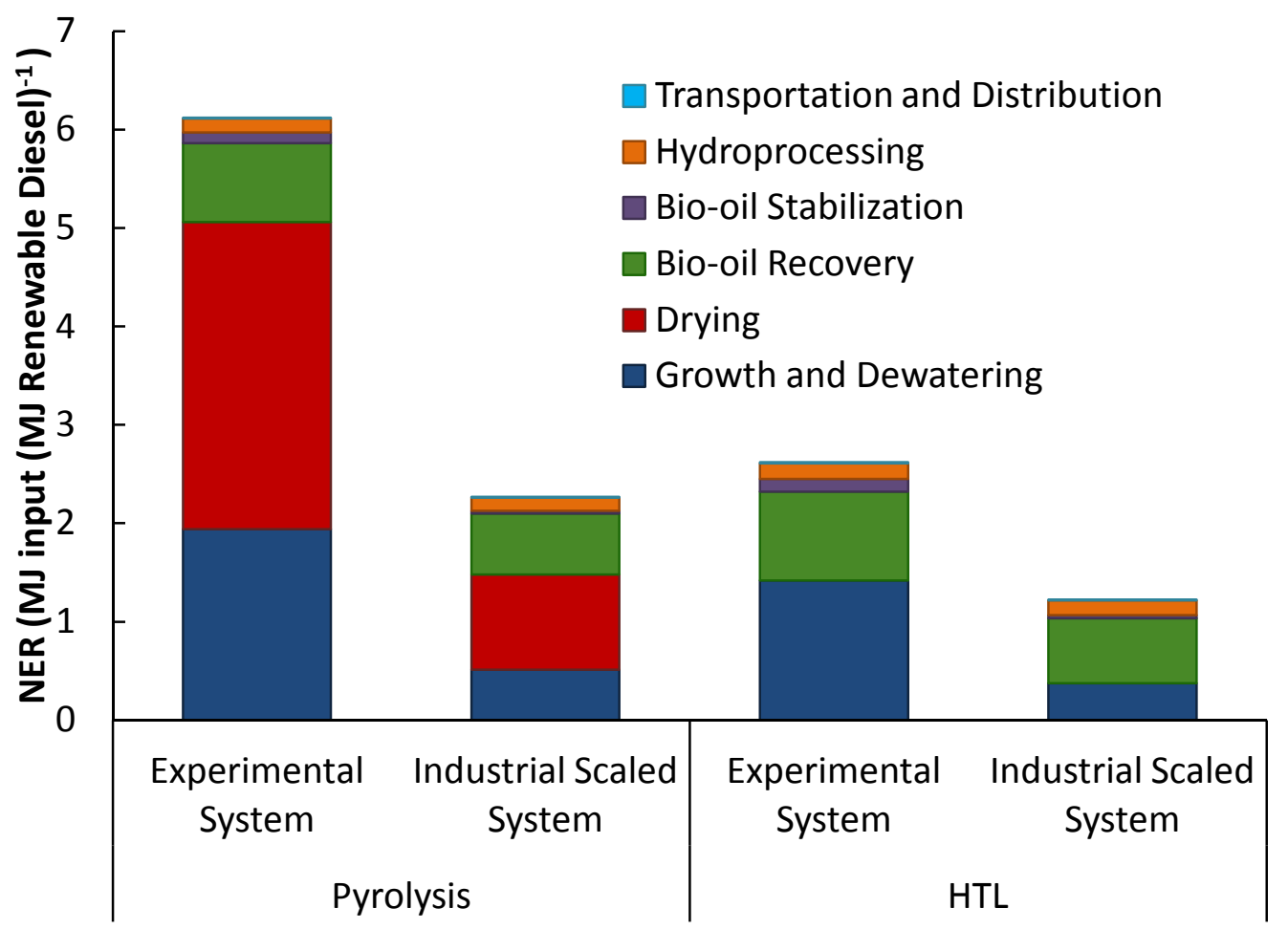

Figure 4: Net Energy Ratio (NER) results for microalgae to renewable diesel with bio-oil recovery through pyrolysis or HTL for small-scale experimental system and the industrial-scaled system.

The results from this study show HTL to be favorable compared to pyrolysis on a system level primarily due to the integration with a wet microalgae slurry (20\% solids), whereas pyrolysis requires dried microalgae ( $80 \%$ solids). The dewater requirements to achieve the percent solids required for HTL conversion facilitates the use of bioflocculation, dissolved air filtration and a centrifuge for removal of water. The pyrolysis pathway requires the remaining water to be removed through thermal methods. Drying of microalgae requires substantial energy, accounting for nearly half (0.97) of the overall NER for the industrial-scale pyrolysis pathway.

The energy flow for the HTL bio-oil recovery processes normalized to 1 unit of energy for the industrial-scale modeling efforts is shown in Figure 5 (A). The HTL process is $55 \%$ efficient in the conversion of embodied feedstock energy to bio-oil. An additional $5.6 \%$ of the sub-process energy is recovered through a heat exchanger and burning of HTL gasses and recycled internally to minimize energy inputs. 
A

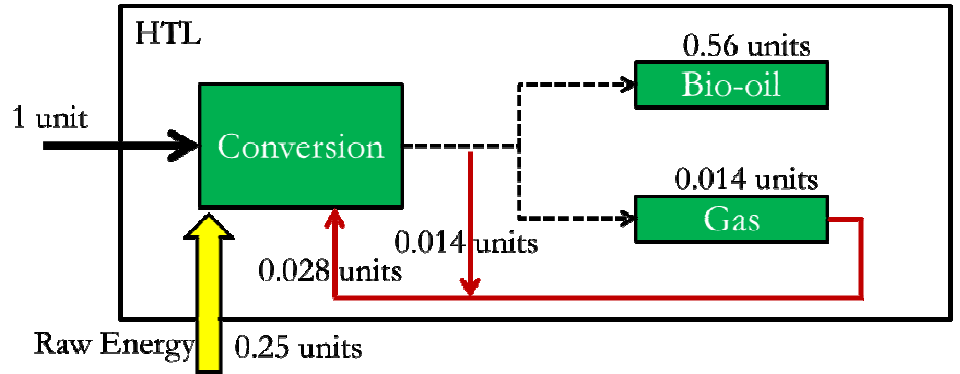

Figure 5: A) Energy flow for the industrial-scale HTL and B) pyrolysis bio-oil recovery sub-processes

372 embodied feedstock energy to bio-oil, Figure 5 (B). The pyrolysis sub-process is

373 integrated into a bio-refinery system allowing for energy recovery through a heat

374 exchanger and combustion of pyrolysis byproducts, char and gasses. Recovered energy

375 accounts for $28 \%$ of the embodied energy in the feedstock, and is used to supplement the

376 energy demand of the drying unit and heating demands in the reactor. Recovered energy

377 helps the overall energetics of the system, but does not negate the energy demands for

378 drying the microalgae biomass or heating in the reactor. Even with energy recovery the

379 combination of the energy demands in the drying unit and pyrolysis reactor are too large

380 for microalgae conversion through pyrolysis to be made energetically favorable.

\subsection{Global Warming Potential}

383 systems for the two thermochemical conversion technologies modeled and compared to

384 conventional diesel, and soybean based biodiesel, Figure 7. The emissions are broken

385 down into process emissions for electrical, heating, and product consumption. Emissions

386 from product consumption are a result of nutrient demands, material losses, and burning

387 of process byproducts, such as char and pyrolysis or HTL gasses. Extending the boundary

388 to well to wheel (WTW) requires the emissions from the combustion of the fuel assumed 
389 here to be $74.1 \mathrm{~g}-\mathrm{CO}_{2-\mathrm{eq}} \mathrm{MJ}^{-1}$ based on $99 \%$ oxidation of the carbon in the fuel with 390 renewable diesel and diesel being equivalent in carbon composition [75].

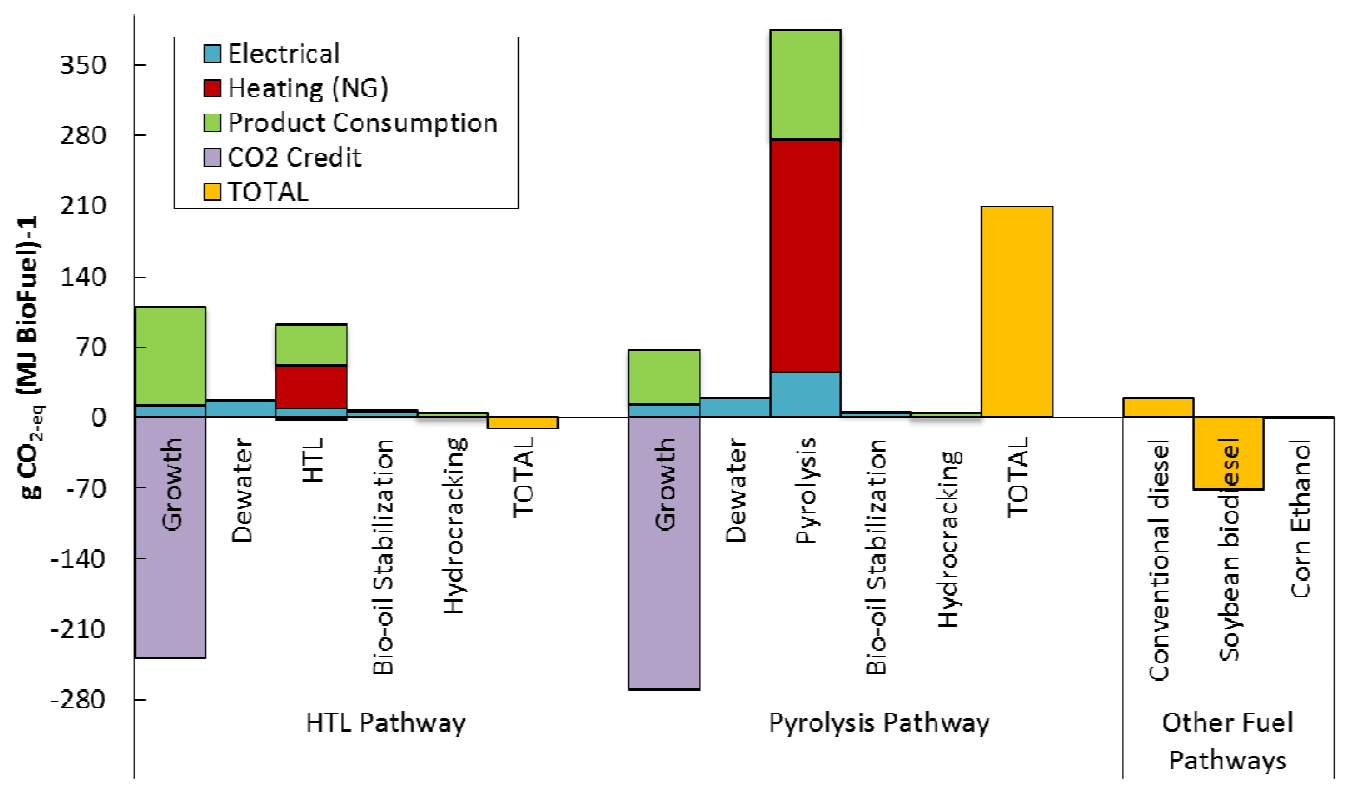

Figure 7: Well to pump GHG emissions for industrial-scale HTL and pyrolysis pathways compared to other traditional fuel pathways [30, 76].

Biomass based biofuel production systems benefit from a carbon credit associated with the uptake of carbon dioxide in the growth phase. The GHG emissions for a WTP systems boundary for the HTL pathway results in net negative $\mathrm{CO}_{2 \text {-eq }}$ emissions of -11.4 $\mathrm{g} \mathrm{CO}_{2 \text {-eq }} \mathrm{MJ}^{-1}$. The aqueous phase from the HTL unit contains ammonium and phosphite, which represent a co-product credit, and is assumed to be recycled and supplement the nutrient requirements for microalgae growth. In terms of GWP, a benefit of bio-oil recovery through HTL results from the processing of a wet microalgae slurry, eliminating the energy and GHG emissions associated with drying. In addition, HTL processing produces a small quantity of combustible gases which are burned to improve the energetics of the system. Compared to conventional diesel, the HTL pathway for the production of renewable diesel results in a 32.5\% reduction in GHG emissions based on a WTW system boundary.

Microalgae conversion through the pyrolysis pathway has two energy intensive processes, microalgae drying and heating in the pyrolysis rector which directly impact the environmental impact of the pathway. The reactor energy is supplemented through burning of pyrolysis byproducts, gas and char, which improves process energetics but are 
410 detrimental to GHG emissions. If burning of pyrolysis char is replaced with natural gas

411 and the produced char is assumed to be land applied, the GHGs for the production of

412 biofuel are reduced from $210 \mathrm{~g} \mathrm{CO}_{2 \text {-eq }} \mathrm{MJ}^{-1}$ to $166 \mathrm{~g} \mathrm{CO}_{2 \text {-eq }} \mathrm{MJ}^{-1}$, with the NER

413 increasing from 2.28 to 2.63 . Using pyrolysis char for alternative purposes would

414 decrease the environmental impact of the pyrolysis pathway, but GHG emissions are still

415 significantly higher than those of conventional diesel and soy biodiesel, and results in an

416 unfavorable increase in the NER. The need of a dry feedstock and energy demands in the

417 reactor for the pyrolysis unit make it difficult to produce an energetically and

418 environmentally favorable renewable fuel. Emissions from microalgae renewable diesel

419 with pyrolysis are high in comparison with conventional diesel and soybean biodiesel.

420

421

422

423

424

425

426

427

428

429

430

431

432

433

434

\subsection{Sensitivity Analysis}

An assessment of the impact of individual parameters was performed on the industrial-scaled system models to better understand inputs that dramatically affect the energetics and environmental impact of the system. Parameters that had a large impact were revisited for accuracy in the scaled-system modeling to increase certainty in results. Results were also used as feedback to experimental systems to identify areas for improvement. Statistical analysis was performed to identify the critical t-ratio based on a 95\% confidence interval (details presented in supplementary information). The results of the sensitivity analysis for the large-scale microalgae to renewable diesel conversion are shown in Figure 8. Sensitivity results are limited to the top 5 input values shown to have the largest impact with full results presented in the supplementary information.
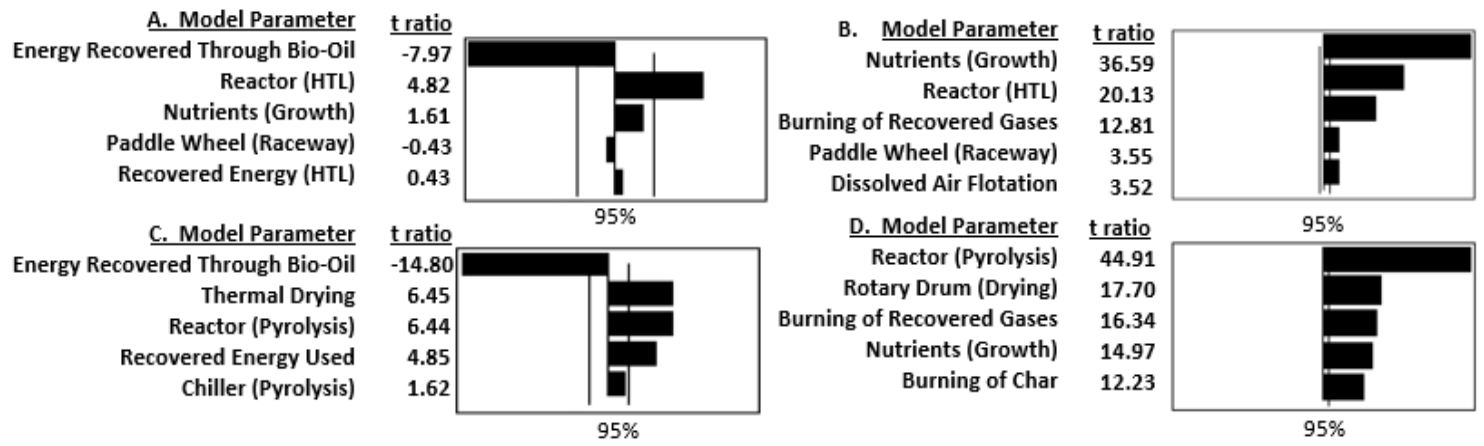

Figure 8: Sensitivity analysis of the WTP system. A) Pyrolysis pathway NER sensitivity, t-critical = \pm 1.73, B) Pyrolysis pathway GHG sensitivity, t-critical $= \pm 1.73$, C) HTL pathway NER sensitivity, tcritical $= \pm 1.75$, D) HTL pathway GHG sensitivity, t-critical $= \pm 1.78$. 
Similarities in the results from the sensitivity analysis for the two thermochemical

436 processes modeled exist as expected. The bio-oil yield represents the functional unit

437 and changes in the yield from the conversion processes will have the largest impact on

438 the system on the metrics of NER. Other inputs shown to be sensitive in the NER

439 sensitivity include reactor energy and recovery of nutrients. Nutrient recycle has been

440 identified as a critical step in the large-scale feasibility of microalgae based biofuel

441 systems [30, 77]. For the pyrolysis pathway drying energy and recovery energy are also

442 sensitive as they have a significant impact on the overall process energetics. Sensitivity

443 in GHG emissions for the respective conversion pathways are shown in Figure 8B and

444 8D. Parameters found to be most sensitive in both conversion methods include emissions

445 associated with conversion and emissions associated with growth in the raceway which

446 are primarily a result of nutrient requirements. In the pyrolysis process drying of

447 microalgae and burning of process byproducts were also found to be sensitive.

$448 \quad 3.4$ Comparison with Literature

449 The current immaturity of the microalgae to biofuels processes has led to the

450 evaluation of a variety of processing technologies on the metrics of GWP. LCA

451 facilitates a holistic comparison of individual sub-processes as the work requires

452 considering the entire process from growth to fuel. A comparison of the results in this

453 study was made to other previously published LCA results, Figure 9. The literature

454 survey was limited to studies that report results based on a system boundary consistent

455 with this study, WTP with the conversion methods used in the various studies

456 highlighted. A similar analysis based on the metric of NER is presented in the

457 supplementary information. 


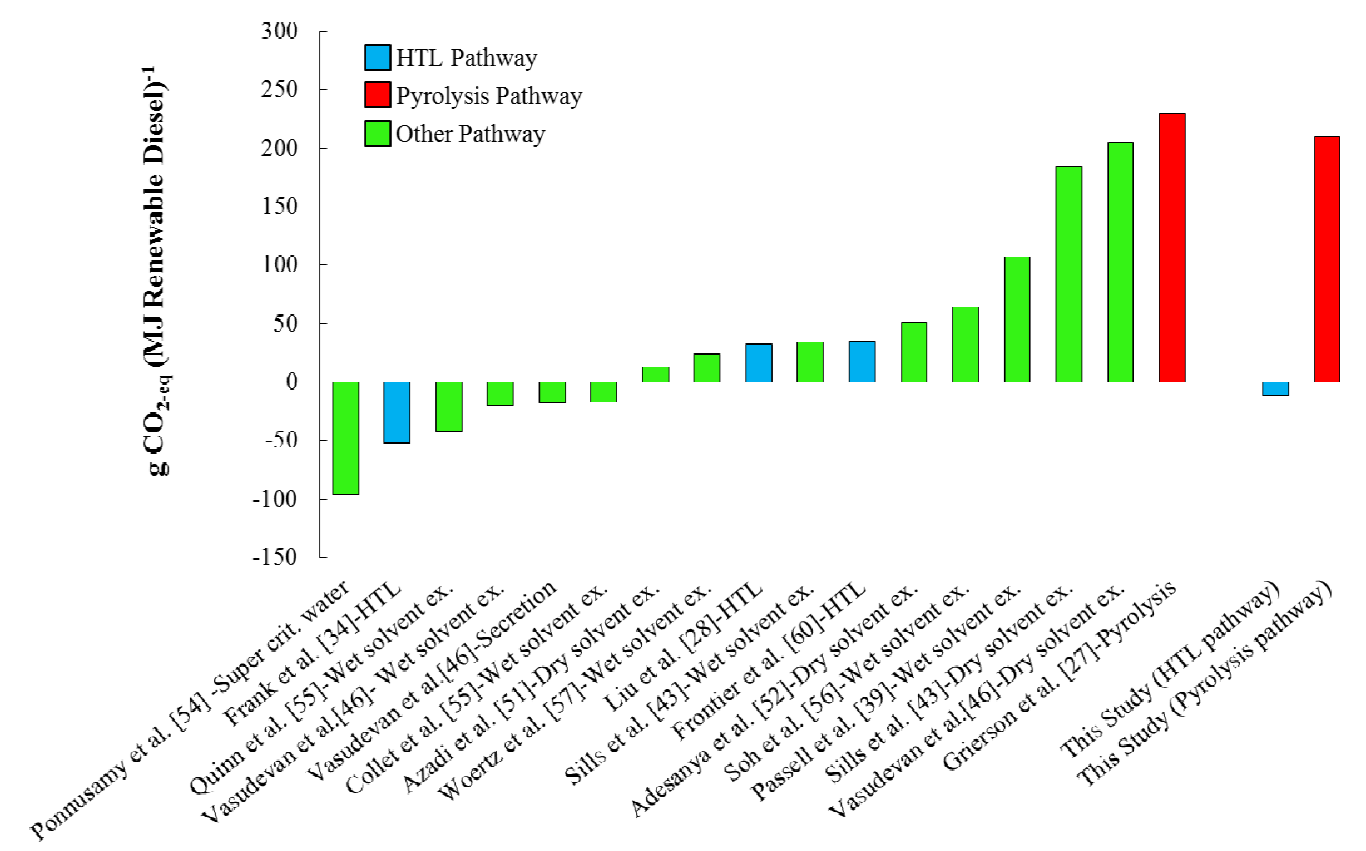

458

459

460

461

462

463

464

465

466

467

468

469

470

471

472

473

474

475

476

477

Figure 9: GHG emissions for microalgae to biofuel with a WTP system boundary as reported in the literature and compared to the results of this study for industrial scale modeling.

The results from the literature survey show a best case WTP GHG emissions for a processes utilizing HTL for conversion of $-52 \mathrm{~g} \mathrm{CO}_{2-\mathrm{eq}} \mathrm{MJ}^{-1}$ reported by Frank et al. [34]

Two other studies examined conversion through HTL, with GHG emission reported at 33 $\mathrm{g} \mathrm{CO}_{2 \text {-eq }} \mathrm{MJ}^{-1}$ by Liu et al.[28] and $35.2 \mathrm{~g} \mathrm{CO}_{2 \text {-eq }} \mathrm{MJ}^{-1}$ by Frontier et al. [60]. Differences in processing pathways and end product can be credited with the differences in results of the various HTL based pathways. Frank et al. [34] report a lower GHG result compared to this study which is attributed to differences in downstream processing following biooil recovery through HTL and higher yield. Stabilization and conversion is done through hydrotreating and hydrocracking of HTL bio-oil, while this study uses a near-critical liquid propane stabilization technique followed by hydroprocessing. At current, the estimated yield from hydroprocessing based on the composition of the bio-oil after biooil stabilization processing in this study is $71 \%$. Optimization of this process is expected to increase the efficiency to $90 \%$ which would improve the environmental impact, further harmonizing results. A direct comparison to Frank et al. [34] is presented in the supplementary information that incorporates restricting the system boundary to growth through HTL processing. The higher GHG emissions in Liu et al.[28] compared to this study are the result of differences in processing pathway. Frontier et al. [60] report a 
478 higher GHG emissions compared to result of this study primarily due to the production of 479 jet fuel compared to renewable diesel in this study. Ultimately, differences in results 480 from the various studies stem from process pathways and assumed HTL performance. 481 Bio-oil production through pyrolysis has been the subject of many studies, but 482 few have evaluated the use of microalgae as the feedstock. In the limited studies that 483 have been performed, differences in pathways require harmonization for direct 484 comparisons. Grierson et al.[27] performs an environmental assessment of a microalgae 485 based biofuel production system incorporating pyrolysis with GHG results reported at $486290.24 \mathrm{~g} \mathrm{CO}_{2-\mathrm{eq}} \mathrm{MJ}^{-1}$ compared to $210 \mathrm{~g} \mathrm{CO}_{2-\mathrm{eq}} \mathrm{MJ}^{-1}$ from this study. The increased 487 GHG emissions in Grierson et al. [27] is attributed to differences in growth architecture, 488 photobioreactor compared to open raceway pond, and water removal through spray 489 drying compared to rotary drum. Large-scale production systems are expected to operate 490 with a drying system similar to the system used in this effort.

\section{Conclusion}

Microalgae is a promising biofuel feedstock due to its ability to grow on nonarable land, does not compete with food, and high yield. LCA currently is being used to assess the large-scale feasibility and environmental impact of alternative processing technologies being explored for processing microalgae as a feedstock into biofuels. This study integrated experimental and literature data for systems engineering model validation to perform an environmental impact and energetic assessment of two different thermochemical conversion technologies, HTL and pyrolysis. Both conversion pathways result in unfavorable NER results with advances in HTL processing expected to improve energetics. Pyrolysis has proven to be an effective way of converting biomass to a

501 biofuel precursor, however on a systems processing level there are challenges associated 502 with microalgae as a feedstock. The biggest challenge comes from drying the microalgae 503 which represents an energy intensive process. The pyrolysis sub-process with microalgae 504 has the potential to be a self-sustaining process, with the ability to recovery nearly two 505 thirds of the total process energy through heat recovery and the burning of byproducts. 506 Excess energy in the pyrolysis process can be used in other processing steps such as 507 drying. The extra energy is limiting to approximatly $20 \%$ of the energy required in the 508 drying process with the remaining energy derived from natural gas. Results from this 
509 study show the pyrolysis pathway is not energetically or environmentally favorable. This

510 is primarily due to microalgae drying dominating the energetics of the process.

\section{Acknowledgments}

$512 \quad$ Funding was provided by U.S. Department of Energy, Energy Efficiency and

513 Renewable Energy, Bioenergy Technology Office, Project Number 2.13.2.6 Utah State

514 University. Research contributions were provided USU bio lab, INL, ASU, and CF

515 Technologies. The authors acknowledge support from Peter Zemke, Alok Shenoy,

516 Buvanish, Brittany Bennion, and Danna Quinn.

\section{References}

518 [1] de Boer K, Moheimani N, Borowitzka M, Bahri P. Extraction and conversion pathways for

519 microalgae to biodiesel: A review focused on energy consumption. J Appl Phycol. 2012;24:1681-

52098.

521 [2] Vardon DR, Sharma BK, Blazina GV, Rajagopalan K, Strathmann TJ. Thermochemical

522 conversion of raw and defatted algal biomass via hydrothermal liquefaction and slow pyrolysis.

523 Bioresour Technol. 2012;109:178-87.

524 [3] Jones S, Davis R, Zhu Y, Kinchin C, Anderson D, Hallen R, et al. Process design and economics

525 for the conversion of algal biomass to hydrocarbons: Whole algae hydrothermal liquefaction and 526 upgrading. Pasific Northwest National Laboratory; March 2014.

527 [4] López Barreiro D, Prins W, Ronsse F, Brilman W. Hydrothermal liquefaction (htl) of

528 microalgae for biofuel production: State of the art review and future prospects. Biomass

529 Bioenergy. 2013;53:113-27.

530 [5] Biller P, Riley R, Ross AB. Catalytic hydrothermal processing of microalgae: Decomposition 531 and upgrading of lipids. Bioresour Technol. 2011;102:4841-8.

532 [6] Biller P, Ross AB. Potential yields and properties of oil from the hydrothermal liquefaction of 533 microalgae with different biochemical content. Bioresour Technol. 2011;102:215-25.

534 [7] Brown TM, Duan P, Savage PE. Hydrothermal liquefaction and gasification of nannochloropsis 535 sp. Energy and Fuels. 2010;24:3639-46.

536 [8] Dote Y, Sawayama S, Inoue S, Minowa T, Yokoyama S-y. Recovery of liquid fuel from

537 hydrocarbon-rich microalgae by thermochemical liquefaction. Fuel. 1994;73:1855-7.

538 [9] Duan P, Savage PE. Hydrothermal liquefaction of a microalga with heterogeneous catalysts. 539 Industrial and Engineering Chemistry Research. 2011;50:52-61.

540 [10] Garcia Alba L, Torri C, Samorì C, Van Der Spek J, Fabbri D, Kersten SRA, et al. Hydrothermal

541 treatment (htt) of microalgae: Evaluation of the process as conversion method in an algae

542 biorefinery concept. Energy and Fuels. 2012;26:642-57.

543 [11] Jena U, Das KC, Kastner JR. Effect of operating conditions of thermochemical liquefaction on 544 biocrude production from spirulina platensis. Bioresour Technol. 2011;102:6221-9.

545 [12] Matsui T, Nishihara A, Ueda C, Ohtsuki M, Ikenaga N, Suzuki T. Liquefaction of micro-algae 546 with iron catalyst. Fuel. 1997;76:1043-8.

547 [13] Minowa T, Yokoyama S-y, Kishimoto M, Okakura T. Oil production from algal cells of 548 dunaliella tertiolecta by direct thermochemical liquefaction. Fuel. 1995;74:1735-8.

549 [14] Ross AB, Biller P, Kubacki ML, Li H, Lea-Langton A, Jones JM. Hydrothermal processing of 550 microalgae using alkali and organic acids. Fuel. 2010;89:2234-43. 
551

552

553

554

555

556

557

558

559

560

561

562

563

564

565

566

567

568

569

570

571

572

573

574

575

576

577

578

579

580

581

582

583

584

585

586

587

588

589

590

591

592

593

594

595

596

[15] Shuping Z, Yulong W, Mingde Y, Kaleem I, Chun L, Tong J. Production and characterization of bio-oil from hydrothermal liquefaction of microalgae dunaliella tertiolecta cake. Energy. 2010;35:5406-11.

[16] Valdez PJ, Dickinson JG, Savage PE. Characterization of product fractions from hydrothermal liquefaction of nannochloropsis sp. And the influence of solvents. Energy and Fuels. 2011;25:3235-43.

[17] Vardon DR, Sharma BK, Scott J, Yu G, Wang Z, Schideman L, et al. Chemical properties of biocrude oil from the hydrothermal liquefaction of spirulina algae, swine manure, and digested anaerobic sludge. Bioresour Technol. 2011;102:8295-303.

[18] Yang YF, Feng CP, Inamori Y, Maekawa T. Analysis of energy conversion characteristics in liquefaction of algae. Resources, Conservation and Recycling. 2004;43:21-33.

[19] Yu G, Zhang Y, Schideman L, Funk TL, Wang Z. Hydrothermal liquefaction of low lipid content microalgae into bio-crude oil. Transactions of the ASABE. 2011;54:239-46.

[20] Zou S, Wu Y, Yang M, Li C, Tong J. Bio-oil production from sub- and supercritical water liquefaction of microalgae dunaliella tertiolecta and related properties. Energy and Environmental Science. 2010;3:1073-8.

[21] Barta K, Ford PC. Catalytic conversion of nonfood woody biomass solids to organic liquids. Accounts of Chemical Research. 2014;47:1503-12.

[22] Corbetta M, Frassoldati A, Bennadji H, Smith K, Serapiglia MJ, Gauthier G, et al. Pyrolysis of centimeter-scale woody biomass particles: Kinetic modeling and experimental validation. Energy \& Fuels. 2014;28:3884-98.

[23] Septien S, Valin S, Dupont C, Peyrot M, Salvador S. Effect of particle size and temperature on woody biomass fast pyrolysis at high temperature (1000-1400 $\left.{ }^{\circ} \mathrm{C}\right)$. Fuel. 2012;97:202-10.

[24] Wannapeera J, Fungtammasan B, Worasuwannarak N. Effects of temperature and holding time during torrefaction on the pyrolysis behaviors of woody biomass. Journal of Analytical and Applied Pyrolysis. 2011;92:99-105.

[25] Mohan D, Pittman CU, Steele PH. Pyrolysis of wood/biomass for bio-oil: A critical review. Energy \& Fuels. 2006;20:848-89.

[26] Di Blasi C. Modeling chemical and physical processes of wood and biomass pyrolysis.

Progress in Energy and Combustion Science. 2008;34:47-90.

[27] Grierson S, Strezov V, Bengtsson J. Life cycle assessment of a microalgae biomass cultivation, bio-oil extraction and pyrolysis processing regime. Algal Research. 2013;2:299-311.

[28] Liu X, Saydah B, Eranki P, Colosi LM, Greg Mitchell B, Rhodes J, et al. Pilot-scale data provide enhanced estimates of the life cycle energy and emissions profile of algae biofuels produced via hydrothermal liquefaction. Bioresour Technol. 2013;148:163-71.

[29] ANL, NREL, PNNL. Renewable diesel from algal lipids: An integrated baseline for cost, emissions, and resource potential from a harmonized model. US Department of Energy Biomass Program; June 2012.

[30] Batan L, Quinn J, Willson B, Bradley T. Net energy and greenhouse gas emission evaluation of biodiesel derived from microalgae. Environmental Science and Technology. 2010;44:7975-80. [31] Beal CM, Hebner RE, Webber ME, Ruoff RS, Seibert AF. The energy return on investment for algal biocrude: Results for a research production facility. BioEnergy Research. 2012;5:341-62. [32] Brentner LB, Eckelman MJ, Zimmerman JB. Combinatorial life cycle assessment to inform process design of industrial production of algal biodiesel. Environ Sci Technol. 2011;45:7060-7. [33] Campbell PK, Beer T, Batten D. Life cycle assessment of biodiesel production from microalgae in ponds. Bioresour Technol. 2011;102:50-6. 
597 [34] Frank E, Elgowainy A, Han J, Wang Z. Life cycle comparison of hydrothermal liquefaction 598 and lipid extraction pathways to renewable diesel from algae. Mitigation and Adaptation 599 Strategies for Global Change. 2013;18:137-58.

600 [35] Jorquera O, Kiperstok A, Sales EA, Embirucu M, Ghirardi ML. Comparative energy life-cycle 601 analyses of microalgal biomass production in open ponds and photobioreactors. Bioresour 602 Technol. 2010;101:1406-13.

603 [36] Khoo HH, Sharratt PN, Das P, Balasubramanian RK, Naraharisetti PK, Shaik S. Life cycle 604 energy and $\mathrm{co} 2$ analysis of microalgae-to-biodiesel: Preliminary results and comparisons.

605 Bioresour Technol. 2011;102:5800-7.

606 [37] Liu X, Clarens AF, Colosi LM. Algae biodiesel has potential despite inconclusive results to 607 date. Bioresour Technol. 2012;104:803-6.

608 [38] Menger-Krug E, Niederste-Hollenberg J, Hillenbrand T, Hiessl H. Integration of microalgae 609 systems at municipal wastewater treatment plants: Implications for energy and emission 610 balances. Environ Sci Technol. 2012;46:11505-14.

611 [39] Passell H, Dhaliwal H, Reno M, Wu B, Ben Amotz A, Ivry E, et al. Algae biodiesel life cycle 612 assessment using current commercial data. J Environ Manag. 2013;129:103-11.

613 [40] Razon LF, Tan RR. Net energy analysis of the production of biodiesel and biogas from the 614 microalgae: Haematococcus pluvialis and nannochloropsis. Applied Energy. 2011;88:3507-14. 615 [41] Sevigné Itoiz E, Fuentes-Grünewald C, Gasol CM, Garcés E, Alacid E, Rossi S, et al. Energy 616 balance and environmental impact analysis of marine microalgal biomass production for 617 biodiesel generation in a photobioreactor pilot plant. Biomass Bioenergy. 2012;39:324-35. 618 [42] Shirvani T, Yan XY, Inderwildi OR, Edwards PP, King DA. Life cycle energy and greenhouse 619 gas analysis for algae-derived biodiesel. Energy \& Environmental Science. 2011;4:3773-8. 620 [43] Sills DL, Paramita V, Franke MJ, Johnson MC, Akabas TM, Greene CH, et al. Quantitative 621 uncertainty analysis of life cycle assessment for algal biofuel production. Environ Sci Technol. 622 2013;47:687-94.

623 [44] Slade R, Bauen A. Micro-algae cultivation for biofuels: Cost, energy balance, environmental 624 impacts and future prospects. Biomass Bioenergy. 2013;53:29-38.

625 [45] Soratana K, Landis AE. Evaluating industrial symbiosis and algae cultivation from a life cycle 626 perspective. Bioresour Technol. 2011;102:6892-901.

627 [46] Vasudevan V, Stratton RW, Pearlson MN, Jersey GR, Beyene AG, Weissman JC, et al.

628 Environmental performance of algal biofuel technology options. Environ Sci Technol.

629 2012;46:2451-9.

630 [47] Williams PJL, Laurens LML. Microalgae as biodiesel \& biomass feedstocks: Review \& analysis 631 of the biochemistry, energetics \& economics. Energy \& Environmental Science. 2010;3:554-90. 632 [48] Xu LX, Brilman DWF, Withag JAM, Brem G, Kersten S. Assessment of a dry and a wet route 633 for the production of biofuels from microalgae: Energy balance analysis. Bioresour Technol. 634 2011;102:5113-22.

635 [49] Yanan Zhang GH, Robert C Brown. Life cycle assessment of the production of hydrogen and 636 transportation fuels from corn stover via fast pyrolysis. Environmental Research Letters.

$637 \quad 2013 ; 8: 1-13$.

638 [50] Frank ED, Han J, Palou-Rivera I, Elgowainy A, Wang MQ. Life-cycle analysis of algal lipid fuels

639 with the greet model. Oak Ridge, TN: Center for Transportation Research, Energy Systems

640 Division, Argonne National Laboratory; 2011.

641 [51] Azadi P, Brownbridge G, Mosbach S, Smallbone A, Bhave A, Inderwildi O, et al. The carbon 642 footprint and non-renewable energy demand of algae-derived biodiesel. Applied Energy. 643 2014;113:1632-44. 
644 [52] Adesanya VO, Cadena E, Scott SA, Smith AG. Life cycle assessment on microalgal biodiesel 645 production using a hybrid cultivation system. Bioresour Technol. 2014;163:343-55.

646 [53] Collet P, Lardon L, Hélias A, Bricout S, Lombaert-Valot I, Perrier B, et al. Biodiesel from 647 microalgae - life cycle assessment and recommendations for potential improvements.

648 Renewable Energy. 2014;71:525-33.

649 [54] Ponnusamy S, Reddy HK, Muppaneni T, Downes CM, Deng S. Life cycle assessment of 650 biodiesel production from algal bio-crude oils extracted under subcritical water conditions. 651 Bioresour Technol. 2014;170:454-61.

652 [55] Quinn JC, Smith TG, Downes CM, Quinn C. Microalgae to biofuels lifecycle assessment653 multiple pathway evaluation. Algal Research. 2014;4:116-22.

654 [56] Soh L, Montazeri M, Haznedaroglu BZ, Kelly C, Peccia J, Eckelman MJ, et al. Evaluating 655 microalgal integrated biorefinery schemes: Empirical controlled growth studies and life cycle 656 assessment. Bioresour Technol. 2014;151:19-27.

657 [57] Woertz IC, Benemann JR, Du N, Unnasch S, Mendola D, Mitchell BG, et al. Life cycle ghg 658 emissions from microalgal biodiesel - a ca-greet model. Environ Sci Technol. 2014;48:6060-8. 659 [58] Luo D, Hu Z, Choi DG, Thomas VM, Realff MJ, Chance RR. Life cycle energy and greenhouse 660 gas emissions for an ethanol production process based on blue-green algae. Environ Sci Technol. 661 2011;44:8670-7.

662 [59] Baliga R, Powers SE. Sustainable algae biodiesel production in cold climates. International 663 Journal of Chemical Engineering. 2010;4:1-13.

664 [60] Fortier M-OP, Roberts GW, Stagg-Williams SM, Sturm BSM. Life cycle assessment of bio-jet 665 fuel from hydrothermal liquefaction of microalgae. Applied Energy. 2014;122:73-82.

666 [61] Richmond A. Handbook of microalgal culture biotechnology and applied phycology. Oxford, 667 UK: Blackwell Science; 2004.

668 [62] Chisti Y. Biodiesel from microalgae. Biotechnol Adv. 2007;25:294-306.

669 [63] Xin L, Hong-ying H, Ke G, Jia Y. Growth and nutrient removal properties of a freshwater 670 microalga scenedesmus sp. Lx1 under different kinds of nitrogen sources. Ecological Engineering. 671 2010;36:379-81.

672 [64] Quinn JC, Catton KB, Johnson S, Thomas HB. Geographical assessment of microalgae 673 biofuels potential incorporating resource availability. BioEnergy Research. 2012;6:591-600.

674 [65] Evodos B.V. Evodos type 102014.

675 [66] Biddy M, Davis R, Jones S, Zhu Y. Whole algae hydrothermal liquefaction technology 676 pathway. Technical Report. 2013.

677 [67] Fan J, Kalnes TN, Alward M, Klinger J, Sadehvandi A, Shonnard DR. Life cycle assessment of 678 electricity generation using fast pyrolysis bio-oil. Renewable Energy. 2011;36:632-41.

679 [68] Chudnovsky Y. High-efficiency, gas-fired drum dryer for food processing applications. In:

680 Commission CE, editor. PIER Industrial/Agricultural/Water End-Use Energy Efficiency

681 Program2011.

682 [69] Shelef G, Sukenik A, Green M. Microalgae harvesting and processing: A literature review. 683 1984. p. Medium: ED; Size: Pages: 70.

684 [70] Agblevor F, Petkovic, L., Bennion, E. P., Quinn, J. C., Moses, J., Newby, D., Ginosar, D. Bio-oil 685 separation and stabilization by supercritical fluid fractionation - final report. In: Report INL, 686 editor.May 2014.

687 [71] United States Environmental Protection Agency. Direct emissions from stationary 688 combustion sources. May 2008.

689 [72] Argonne National Laboratory. Greet 2013 model. 2013. 
690 [73] Solomon S, Qin D, Manning M, Chen Z, Marquis M, Averyt KB, et al. Contribution of working 691 group $i$ to the fourth assessment report of the intergovernmental panel on climate change, 2007 692 Cambridge University Press; 2007.

693 [74] Murphy D, Hall CS, Powers B. New perspectives on the energy return on (energy)

694 investment (eroi) of corn ethanol. Environment, Development and Sustainability. 2011;13:179-

695202

696 [75] United States Environmental Protection Agency. Average carbon dioxide emissions resulting 697 from gasoline and diesel fuel. 2005.

698 [76] Wang M, Han J, Dunn JB, Cai H, Elgowainy A. Well-to-wheels energy use and greenhouse 699 gas emissions of ethanol from corn, sugarcane and cellulosic biomass for us use. Environmental 700 Research Letters. 2012;7:045905.

701 [77] Pate R, Klise G, Wu B. Resource demand implications for us algae biofuels production scale702 up. Applied Energy. 2011;88:3377-88.

703 
Click here to download e-component: Supplementary Material_v2.pdf

.

.

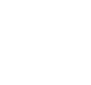

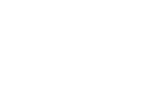

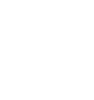

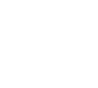

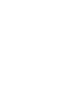

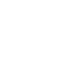

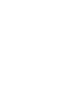

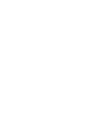
. 列 . . . . . . . . . 